\title{
Manfred Walther
}

\section{Kommunalismus und Vertragstheorie.}

\author{
Althusius - Hobbes - Spinoza - Rousseau \\ oder \\ Tradition und Gestaltwandel einer politischen Erfahrung
}

\section{Fragestellung und Aufbau der Untersuchung}

1.1 Der Kommunalismus ist diejenige Form politischer Organisation zusammenlebender Menschen, in der die Regeln des Zusammenlebens und die alle betreffenden Entscheidungen unter aktiver Mitwirkung aller (communis) zustandekommen, die also Selbstorganisation der Betroffenen ist. ${ }^{1}$ Der Kommunalismus gründet in der Erfahrung, daß die gemeinsame, einvernehmliche Ausübung der dem einzelnen eigenen Handlungsmacht die Chance eröffnet, als niederdrückend empfundenen Formen personaler Herrschaft widerstehen und sich diesen gegenüber behaupten zu können. Es ist also die Erfahrung der Möglichkeit erfolgreichen gemeinsamen (communis) Betreibens der Angelegenheiten des eigenen Lebensbereiches auch gegen vorhandene personale Herrschaft, welche dem Kommunalismus als einer der Formen der Emanzipation aus dem „Feudalismus" zugrunde liegt.

Das Problem, das sich für den Kommunalismus als verallgemeinerungsfähige Form politischer Organisation stellt, ist ein doppeltes:

- Wie läßt sich diese als Negation bestehender Herrschaftsverhältnisse entstandene Form auf Dauer stellen, auch wenn das auslösende Gegenbild zum Verschwinden gebracht worden ist?

- In welcher Intensität und inhaltlichen Breite läßt sich eine solche Einheit des

1 Peter Blickle formuliert, im Sinne einer Arbeitsdefinition: „Kommunalismus heißt..., daß die Organisation gemeinschaftlicher, alltäglicher Belange (ausgedrückt in Satzungshoheit, Administration und Rechtspflege), die Friedenswahrung nach innen und außen und die aus beiden resultierenden Rechtsnormen als autochthone Rechte einer Gemeinde von allen Mitgliedern in gleicher Berechtigung und Verpflichtung wahrgenommen werden. Berechtigung und Verpflichtung erwachsen aus der selbstverantwortlichen Arbeit als Bauer und Handwerker im genossenschaftlichen Verband." Peter Blickle, Kommunalismus, Parlamentarismus, Republikanismus, in: Historische Zeitschrift 242 (1986) 529-536 (535). 
Wollens und Tuns durchhalten, wenn der für die Selbsterhaltung der Gemeinde konstitutive Bereich sozialer etc. Interaktionen sich ausweitet und zugleich die gesellschaftlichen Verhältnisse sich ökonomisch, sozial, religiös etc. immer weiter ausdifferenzieren?

Meine These ist, daß der Kommunalismus nur funktionsfähig ist und bleibt, solange die Bedrohung durch personale Herrschaft anhält, daß er also wesentlich Reaktion auf derartige Verhältnisse ist; und daß er zudem auf eine überschaubare und von allen geteilte Lebenswelt begrenzt bleibt. Entfallen diese Bedingungen, so verfällt er entweder oder regrediert zu einer gesinnungsdrückenden, sich gegen gesellschaftliche Dynamik stellende Zwangsidentität, oder er verknöchert.

1.2 Daraus ergeben sich zwei Leitgesichtspunkte, unter denen ich ausgewählte Theoretiker der Politik des 17. und 18. Jahrhunderts unter dem Leitgesichtspunkt „Kommunalismus“ befragen werde:

- Wo und in welcher Gestalt lassen sich Reflexe der Erfahrungen des Kommunalismus von der Überlegenheit kooperativer gegenüber herrschaftlicher politischer Organisation in diesen Theorien nachweisen?

- Auf welche Weise reagieren diese Theoretiker auf die Tatsache zunehmender gesellschaftlicher Differenzierung und Individualisierung und die damit verknüpfte „Verdünnung" gemeinsamer Lebensformen und Überzeugungen?

Bezogen auf den Staatsbildungsprozeß des untersuchten Zeitraumes geht es, politiktheoretisch gesprochen, um die Möglichkeit der Verbindung von Demokratisierung der politischen Willensbildung mit der Ausübung - immer größere soziale und territoriale Einheiten umfassender - gesamtstaatlicher Souveränität. Während man sofort sieht, daß die Demokratisierungsbestrebungen einen direkten Anschluß an die kommunalistische Erfahrung darstellen, erscheint es problematisch, wie angesichts fortschreitender gesellschaftlicher Differenzierung in Religion, Ökonomie, Wissenschaft etc. politische Einheit noch im Sinne politischer Selbstorganisation von allen geteilter Lebenswelten funktionieren soll. Anders gesagt: Wie das Erfordernis einer mit "Letztentscheidungskompetenz" ausgestatteten personalen Herrschaft mit der Demokratisierung der politischen Willensbildung so verknüpft werden kann, daß beides gewährleistet ist. Im Schnittpunkt von Demokratie als Selbstherrschaft und Souveränität als personaler Herrschaft steht das Problem des Widerstandsrechts: Widerstandsrecht ist, systematisch betrachtet, diejenige Rechtsfigur, welche den Vorrang des gemeinsamen Willens der Bürger angesichts der für diese dysfunktionalen Folgen einer Verselbständigung personaler Herrschaft im Konfliktfall wiederherstellen soll; das Widerstandsrecht ist der Versuch, dem Auseinanderfallen von Geltung des gemeinsamen Willens der zusammenlebenden Bürger und Wirksamkeit dieses Willens im Medium personaler Dispositionsgewalt zu begegnen.

1.3 Die grundlegenden Figuren der Bildung und Ausübung eines gemeinsamen Willens der sich zusammenschließenden Bürger sind der Vertrag, der Bund und die Beauftragung. 
- Der Vertrag ist diejenige Figur, in der sich mehrere Willen wechselseitig binden, d. h. bezüglich der Willensausübung gegenüber den Vertragspartnern festlegen und so Erwartungssicherheit begründen. Dabei sind dem Vertragsinhalt nach zwei Formen zu unterscheiden:

- Im Austauschvertrag verpflichten sich die Vertragspartner dazu, wechselseitig füreinander, sei es gleichzeitig, sei es nacheinander, bestimmte Leistungen zu erbringen.

- Im Kooperationsvertrag verpflichten sie sich zur gemeinschaftlichen Ausführung bestimmter Handlungen ${ }^{2}$.

- Der Bund ist ein „willentliche(r) Zusammenschluß... zur Verwirklichung einer bestimmten gemeinsamen Lebensweise, die allen als unbedingter Z $Z$ weck des individuellen und gemeinsamen Handelns gilt ${ }^{\text {" }}$. Der Bund kann als eine spezifische Gestalt des Kooperationsvertrages verstanden werden, in dem sich die Vertragspartner hinsichtlich der Gesamtgestalt ihrer Lebensverhältnisse an den Konsens binden.

Das Verhältnis zwischen Vertrag und Bund ist also dieses, daß jeder Bund ein Vertrag ist, aber nicht umgekehrt. Verträge, in denen die Leistung mindestens eines der Vertragspartner erst später erfolgen soll, enthalten immer ein Moment der Treue und berühren sich somit mit dem Bundesgedanken.

- Die Delegation der Ausführung bestimmter gemeinsam gewollter Handlungen an einzelne oder Gruppen einschließlich der Kompetenz, über die dazu erforderlichen Mittel zu disponieren, ist die Beauftragung.

Der Kommunalismus ist, insofern er ein willentlicher Zusammenschluß zur gemeinsamen Lebensführung ist, ein Bund, der mit der Vergabe von Aufträgen durchaus kompatibel ist.

1.4 Mit den drei Auswahlkriterien, Demokratisierung, Souveränität und Widerstandsrecht, werde ich im folgenden die kontraktualistisch relevanten Aspekte der politischen Theorien oder Philosophien von Althusius (Deutschland/Niederlande) (3.), Hobbes (England) (4.), Spinoza (Niederlande) (5.) und Rousseau (Frankreich) (6.) daraufhin untersuchen, ob, inwieweit und in welcher Form in ihnen die kommunalistische Erfahrung fortwirkt und ob und in welcher Weise sie sich dabei des Vertragsgedankens bedienen. Alle vier haben ihre mental-kulturelle Prägung im reformatorischen „Milieu“, drei von ihnen vornehmlich im reformierten, erfahren ${ }^{4}$. Und drei von ihnen verarbeiten Erfahrungen mit revolutionären

2 Wolfgang Kersting, Die politische Philosophie des Gesellschaftsvertrages (Darmstadt 1994) 217, unterscheidet im Blick auf den staatstheoretischen Kontraktualismus die beiden Momente der "Autoritäts- und Herrschaftslegitimation durch freiwillige Selbstbindung" und die "rationale... Kooperationsform... der Individuen“.

3 Ludwig Siep, Vertragstheorie - Ermächtigung oder Kritik von Herrschaft?, in: Furcht und Freiheit: Leviathan-Diskussion 300 Jahre nach Thomas Hobbes, hrsg. von Udo Bermbach, Klaus-M. Kodalle (Opladen 1982) 129-145 (131). Die Unterscheidung von Vertrag, Bund und Beauftragung, die ich hier einführe, ebd.

+ Bei Spinoza kommt freilich noch die der sonstigen Entwicklung vorauseilende Marranenerfahrung hinzu, also die Erfahrung der einem Wechselbad von Zumutungen verschiedener 
politischen Umbrüchen (Niederlande und England), einer, nämlich Rousseau, bereitet einen solchen Traditionsbruch theoretisch vor.

Dem reformatorischen Hintergrund in Gestalt der reformierten Tradition politischer Theologie, den diese Autoren mehr oder weniger intensiv teilen oder zumindest kennen, ist ein eigener Artikel in diesem Band gewidmet. Deshalb schicke ich hier nur eine kurze, auch als Kontrast dienende, Skizze der politischen Theologie Luthers voran (2.).

Am Schluß möchte ich versuchen, einen strukturvergleichenden Rückblick auf die behandelten Autoren mit einem Ausblick zu verbinden, und zwar auf die gegenwärtige Gestalt, in der das kommunalistische Problem virulent ist, nämlich auf den Kommunitarismus (7.).

\section{Luthers politische Theologie}

Luthers Grundvorstellung über die Organisation des Zusammenlebens der Menschen ist die der personalen Herrschaft: So wie Gott über die Gläubigen - in Liebe - herrscht, so herrscht die „Obrigkeit" - mit Recht und Schwert, aber auch mit Klugheit und Billigkeit, wenn's geht - über die Untertanen. Und die - durch den unterschiedlichen, ja im entscheidenden Punkt gegensätzlichen Charakter des jeweils herrschenden Gesetzes, des Liebesgesetzes auf der einen, des Gesetzes der Selbstbehauptung von im besten Falle rationalen Egoisten auf der anderen Seite (Zwei-Reiche-Lehre) nicht beeinträchtigte - Isomorphie göttlicher und weltlicher personaler Herrschaft bildet die zweifelsfreie Grundlage der gesamten politischen Lehre; das Gefälle zwischen göttlicher und weltlicher Herrschaft kommt dann, zusätzlich zu der unterschiedlichen Qualität des jeweils herrschenden Gesetzes, darin zum Ausdruck, daß die göttliche Herrschaft auf die obrigkeitliche ausstrahlt, sie „heiligt“. Der Gedanke der politischen Selbstorganisation der Menschen als Bürger ist Luthers Denken, und zwar vor allem aus theologischen Gründen - Folgelast des Abfalls von Gott -, so fremd, wie er als Gleichheit und Priestertum aller Gläubigen für Luthers Gemeindebegriff konstitutiv ist - so als hätte er alle autoritären Züge, die aus seinem Gemeindebegriff gebannt sind, umso stärker in seinem Begriff der politischen Einheitsbildung akkumuliert. Die kommunalistische Erfahrung, daß politische Selbstorganisation der Bürger eines Gemeinwesens möglich ist, und die der Luther lesende „gemeine Mann“ aus seinen frühen Schriften als politische Verheißung glaubte entnehmen zu können, ist für Luther, vor allem in Gestalt der Münzerschen Theologie, theologisch disqualifiziert und kann sich deshalb auch politisch nur verheerend auswirken. In der Zwei-ReicheLehre Luthers liegen so die Wurzeln einer Ausdifferenzierung, hier in Form einer

positiver Religionen und zugleich dem Rationalismus der spanischen Spätscholastik ausgesetzten spanisch-portugiesischen Juden. Vgl. dazu knapp Manfred Walther, Negri on Spinoza's Political and Legal Philosophy, in: Spinoza: Issues and Directions, ed. by E. Curley, P.-F. Moreau (Brill's Studies in Intellectual History 14, Leiden u. a. 1990) 286-297 (289), sowie ausführlich Yirmiyahu Yovel, Spinoza: Das Abenteuer der Immanenz (Göttingen 1994) I. Teil: Der Marrane der Vernunft. 
schroffen Entgegensetzung, von Moral und Sittlichkeit innengeleiteter Lebensführung aus der Quelle des Evangeliums und einer positiven Zwangsordnung schon fast rechtspositivistischer Prägung. Eine Verbindung zwischen beiden Reichen bildet bei ihm, abgesehen von dem guten und gerechten Herrscher, einem freilich „seltenen Vogel“, nur das Wirken und ggf. das zeugnishafte Leiden der Christen, die den Gehorsam gegen den Glaubenszwang des Fürsten verweigern und die damit und nur damit - in einer Haltung des theoligical disobedience - widerstehen, aber die politische Ordnung nicht infrage stellen, vielmehr durch das Zeugnis geduldigen Ertragens obrigkeitlicher Strafe das Reich Gottes zur Rechten in seiner Kraft in das Reich Gottes zur Linken hineinleuchten lassen. Die Glaubens- und Gewissensfreiheit, die Luther mit zahlreichen Argumenten in den Frühschriften als unabdingbar nachweist, geht freilich später, als das Territorialkirchentum sich zu formieren beginnt, fast gänzlich verloren.

Damit ist die Idee der kommunalen Selbstorganisation und überhaupt der politischen Selbstorganisation im Wirkungsbereich der lutherischen Reformation als utopisches Aufbegehren des sündigen Menschen gegen Gottes Erhaltungsordnung gebrandmarkt, und von den traumatischen Erfahrungen der politischen und der religiösen Disqualifikation aller solcher Versuche in den „Bauernkriegen“ erholt sich das politische Bewußtsein der Deutschen für Jahrhunderte nicht mehr. Ganz anders aber verläuft die Entwicklung dort, wo man an die reformierte Lehrbildung anschließt.

\section{Johannes Althusius oder die kontraktualistische Modifikation des Aristotelismus}

3.1 Johannes Althusius (ca. 1557-1638), in Basel und Genf ausgebildeter promovierter Jurist, veröffentlicht, wohl auch im Hinblick auf die 1604 angetretene und bis ans Lebensende ausgeübte Tätigkeit als Syndikus der Stadt Emden, 1603 seine „Politica methodice digesta“, die bis 1614 zwei weitere, $z$. T. erheblich überarbeitete und erweiterte Auflagen erlebt ${ }^{5}$, dann aber bald in Vergessenheit gerät und erst von Otto Gierke neu entdeckt wird ${ }^{6}$ und seitdem, in zunehmendem Umfang, die Aufmerksamkeit der theoriegeschichtlichen Forschung gefunden hat ${ }^{7}$.

${ }^{5}$ Zitiert wird nach dem 2. Nachdruck der Ausgabe Herborn 1614, Aalen 1981, und zwar nach Kapitel/Randnummer.

${ }^{6}$ Otto von Gierke, Johannes Althusius und die Entwicklung der naturrechtlichen Staatstheorien: Zugleich ein Beitrag zur Geschichte der Rechtssystematik (Aalen ${ }^{6} 1968$; zuerst 1880). Gierke war dabei von dem Interesse geleitet, auch in Deutschland einen politischen Denker in der Zeit zwischen Bodin und der deutschen Aufklärung ausfindig zu machen, der einen Beitrag zur modernen, d.i. zur gesellschaftsvertraglich konzipierten, Staatstheorie geleistet hat. Althusius erfüllte - Gierke zufolge - nicht nur diese Erwartung, sondern erwies sich darüber hinaus als bedeutender Protagonist des von Gierke favorisierten Genossenschaftsprinzips der Staatsbildung.

7 Hingewiesen sei nur auf den Sammelband Karl-Wilhelm Dabm, Werner Krawietz (Hrsg.), Politische Theorie des Johannes Althusius (Rechtstheorie, Beiheft 7, Berlin 1988) im folgenden zit. als Politische Theorie sowie auf die, die mögliche Relevanz des Althusius für Probleme eines föderativen Staatsaufbaus untersuchenden Arbeiten von Thomas $O$. Huegelein, 
3.2 Althusius, der um die Ausarbeitung einer gegenüber Theologie, Jurisprudenz und Philosophie selbständigen politischen Wissenschaft bemüht ist ${ }^{8}$, greift dafür auf die seit Thomas von Aquin wieder bekannte und genutzte aristotelische Politik zurück. Zugleich aber verarbeitet er die für den Protestantismus reformierter Prägung katastrophale Erfahrung der Bartholomäusnacht in Frankreich (1572) in der Weise, daß er, entgegen dem Impetus der Zwei-Reiche-Lehre der Reformatoren der ersten Generationen, von vornherein jede mögliche politische Einheit konfessionell, d. h. als einen das Volk als Volk Gottes konstituierenden Bund mit Gott konzipiert ${ }^{9}$ und damit die der reformierten Tradition eigentümliche Tendenz, Glaubens- und Bürgergemeinde als innigst verbunden zu sehen, noch verstärkt. Das bietet zugleich die Möglichkeit, die kommunalistische Erfahrung, jetzt im Gegenüber zu der noch recht neuen Bodinschen Lehre von der gesetzesüberhobenen Fürstensouveränität, zur Geltung zu bringen. Denn der Bundesgedanke der alttestamentarischen Überlieferung hat eine seiner Sinnspitzen darin, mit dem Herausstellen der Königsherrschaft Gottes über sein Volk zugleich jede ursprüngliche, nicht vom Volk selber eingesetzte Herrschaft von Menschen über Menschen als frevelhafte Imitation der Gottesherrschaft theologisch zu delegitimieren ${ }^{10}$. Spinoza wird das später, kritisch gegen die monarchistischen Bestrebungen der calvinistischen Predikanten seiner Zeit, so auf den Begriff bringen, daß die Theokratie eine ihrer selbst noch nicht bewußte Form der Selbstherrschaft des Volkes ist $^{11}$.

3.3 Politische Wissenschaft ist nach Althusius die Kunstlehre (ars) von Zielen, Ursachen, Form und Struktur von „Vereinigungen zum gemeinsamen Leben“ (consociationes symbiotikae), die er, gemäß der aristotelischen Lehre von den vier causae, folgendermaßen entfaltet:

Ziel (finis) der consociationes symbiotikae ist die Beförderung des zum Leben der Mitglieder Erforderlichen (necessaria) und Nützlichen (utilia), und zwar im Hinblick auf das gesamte, Geist und Körper umfassende, Leben - in erster Linie für

Johannes Althusius: eine „alternative“ Institutionentheorie der Frühen Neuzeit, in: Politische Institutionen im gesellschaftlichen Wandel, hrsg. von G. Göhler, K. Lenk, H. Münkler, M. Walther (Opladen 1990) 203-235 und ders., Sozialer Föderalismus: Die politische Theorie des Johannes Althusius (Berlin 1991).

8 Vgl. dazu vor allem das Vorwort zur 1. Aufl. der Politica von 1603.

9 Wilhelm Schmidt-Biggemann, Althusius" politische Theologie, in: Politische Theorie (wie Anm. 7), 212-231 (218-220).

10 Zur innerjüdischen Kontroverse über die Legitimität königlicher Herrschaft und deren Niederschlag in den alttestamentarischen Schriften vgl. Peter Weber-Schäfer, Heil und Herrschaft bei den Juden: Könige und Propheten, in: Bürgerreligion und Bürgertugend: Debatten über die vorpolitischen Grundlagen politischer Ordnung, hrsg. von Herfried Mïnkler (Baden-Baden 1995) (im Druck).

11 Baruch de Spinoza, Theologisch-politischer Traktat (Hamburg 1976) zit.: TTP, nach Kapitel: Seitenzahl; 17: 254-255. S.a. Manfred Walther, Institution, Imagination und Freiheit bei Spinoza: Eine kritische Theorie politischer Institutionen, in: Politische Institutionen 1990 (wie Anm. 7), 246-275 (255-260). 
den Geist, d. h. „zur Einrichtung und zur Unterrichtung der Untertanen zur wahren Erkenntnis Gottes und seiner Verehrung; und zur Anordnung von Pflichten, die dem Nächsten gegenüber verrichtet werden müssen, schließlich zur Berichtigung schlechter Sitten und von Irrtümern“. Es geht also um die "heilsame Erkenntnis der res sancta, justa et utilia" (I/15). Ein politeuma ist Gemeinschaft des Rechts, der Einrichtung und Verwaltung des Gemeinwesens, der Ordnung und Verfassung der Bürgerschaft, und alle Mitglieder partizipieren an den so beschriebenen und besorgten gemeinsamen Gütern (II/5.6).

Ursache (causa efficiens) sind auf der jeweiligen Vergemeinschaftungsstufe die Mitglieder: bei den einfachen und privaten consociationes die Individuen (II/3), welche sowohl die nicht gewillkürte, sondern notwendige Gemeinschaft der Ehe und des ganzen Hauses, als auch die gewillkürten Kollegien, also die Berufsstände bilden, die daher auch wieder aufgelöst werden können (IV/1); bei den gemischten und öffentlichen und, weil nicht mehr funktional spezifiziert, auch allgemeinen, die ein politeuma bilden, die jew eils vorangehenden sozialen Einheiten(V/2). Ohne eine so begründete Gemeinschaft des Rechts und der - ausübenden - Gewalt (V/5) ist die Ansammlung von einzelnen nur eine multitudo, kein populus (V/4).

Die Form der Lebensgemeinschaft ist die Übereinstimmung (consensus), kraft der die symbiotisch Verbundenen „ein Herz und eine Seele“ (anima E cor unum) sind (!), die "dasselbe wollen, tun, nicht wollen, zum gemeinsamen Nutzen der Verbundenen " (II/8); und so verkörpert (repraesentat) die consociatio "oft" eine Person und wird als solche genommen.

Die Struktur der consociatio ist die der Herrschaft: eines Herrschenden oder eines Kollegiums von Herrschenden, dem oder denen die anderen Mitglieder als einzelne (singuli) zum Gehorsam verpflichtet, aber als Gesamtheit (universi) übergeordnet sind.

Mehrere politeumata der ersten Stufe, Städte und Dörfer, schließen sich zu einer Provinz zusammen, mehrere Provinzen zu einer consociatio „universalis, publica, major" (IX/1), die als „politia, imperium, regnum, respublica" bezeichnet wird und herrschaftlich verfaßt ist, weil anders eine „vita pia $\mathcal{E}$ justa " nicht möglich ist (IX/3).

Für das Verhältnis der stufenweise aufeinander folgenden politeumata gilt, daß

- ihre Mitglieder nicht Individuen, sondern zunächst die privaten, sowohl die notwendigen wie die gewillkürten (spontanaea), Gemeinschaften, dann mit diesen auch die politeumata niedrigerer Stufe sind, so daß es sich um consociationes mixtae handelt (IX/3);

- die Übertragung von Funktionen an die jeweils höhere Gemeinschaft nach dem Subsidiaritätsprinzip erfolgt.

3.4 Der Modus des Zusammenschlusses ist das pactum, und zwar entweder das ausdrückliche oder das stillschweigende, kraft dessen sich die Symbioten wechselseitig verpflichten (I/2). Das Recht, dessen Einheit und gemeinschaftliche Verwaltung die Einheit des politeuma konstituiert (IX/3), ist das im Dekalog offenbarte göttliche Recht, das mit dem Naturrecht identisch ist. 
$\mathrm{Zu}$ seiner Verwaltung (administratio) setzt das politeuma, hier am Beispiel der consociatio universalis publica major illustriert, also das in der geschilderten Weise gegliederte Volk, das eigentlicher Inhaber der Herrschaft ist (IX/5), Verwalter ein (IX.4). Akteure dieser Einsetzung sind die das Volk - „im Sinne ständischer Identitätsrepräsentation "12 - vertretenden Ephoren ${ }^{13}$. Die Einsetzung geschieht coram Deo, d. h. im Rahmen eines Bundes des Volkes mit Gott, vor dem sich somit alle Beteiligten zur getreulichen Ausübung ihrer Funktionen verpflichten und dessen Sanktionsgewalt sie sich damit unterstellen (28/15.17). Diese Bestellung des summus magistratus zur Verwaltung der gemeinsamen Angelegenheiten des politeuma erfolgt nun ihrerseits in der Form eines Vertrages (contractus) in der Weise, daß das Herrschaftsrecht von den Ephoren unter von diesen festgesetzten Bedingungen, den leges fundamentales, an den Magistrat übertragen wird, während sich umgekehrt das Volk durch die Ephoren zum Gehorsam gegenüber dem Magistrat verpflichtet (XIX/23) ${ }^{14}$. Während die Magistrate als Beauftragte des populus zur Erfüllung der übertragenen Aufgaben fungieren - und dazu gehört auch die situationsspezifische Konkretisierung des natürlichen = göttlichen Rechts -, haben die Ephoren die Aufgabe, die Magistrate auf Einhaltung dieser Regeln und allgemein auf funktionsgerechte Erfüllung dieser Aufgaben hin zu kontrollieren.

Verstoßen die Inhaber der Magistratur obstinatè, violatà fide E religione jurisjurandi gegen ihre Kompetenzen, verhalten sie sich also tyrannisch (XXXVIII/3), so setzen sie sich damit als pactum und contractum brechend zu privati herab (XXXVIII/37) und können von den Ephoren, aber nur von diesen - und hier subsidiär in absteigender Linie - zur Rechenschaft gezogen, abgesetzt, ja im Extremfall getötet werden.

3.5 Althusius verbindet im nachreformatorischen Zeitalter noch einmal die aristotelische Konzeption einer stufenweise sich herausbildenden teleologisch geprägten Vergesellschaftung, die reformierte Doppelgestaltung von Glaubens- und Bürgergemeinde und die kommunalistische Erfahrung der Kraft und Stärke einer Willens- und Gesinnungsgemeinschaft der Lebensführung zu einer kunstvollen (ars) Einheit, die aber sowohl aufgrund der Heterogenität der synthetisierten Elemente als auch aufgrund der Substantialität der geforderten Einheitsbildung dem sich verstärkenden Druck gesellschaftlicher Differenzierung und damit auch der Ausbildung religiöser, wissenschaftlicher und ökonomischer Individualität nicht lange gewachsen ist und daher nicht, wie Gierke meinte, den auch später nicht

12 Kersting, (wie Anm. 2), 224, im Anschluß an Hasso Hofmann, Repräsentation: Studien zur Wort- und Begriffsgeschichte von der Antike bis ins 19. Jahrhundert (Berlin 1994) 358-373.

13 Hier rezipiert Althusius also das in der calvinistischen Tradition entwickelte Schema einer doppelten Führung, durch die Ephoren einerseits, durch die Magistrate andererseits.

14 Vgl. Kersting, (wie Anm. 2), 224. Das Problem, wie denn das Volk mit dem zukünftigen Magistrat als einem seiner Bestandteile einen solchen bedingten Austauschvertrag schließen kann, wird Hobbes später konsequent in der Weise lösen, daß bei ihm der Herrscher nicht Vertragspartner, sondern nur Begünstiger des Vertrags ist. 
übertroffenen Gipfel moderner Staatstheorie bildet, sondern in einer „Sackgasse der Evolution" endet und daher später nicht mehr anschlußfähig ist ${ }^{15}$.

a) Althusius und der neuzeitliche Kontraktualismus

- Zunächst ist anzumerken, daß Althusius zwar mit dem Vertragsgedanken, wie er der modernen Gesellschaftsvertragstheorie zugrunde liegt, arbeitet, aber die Subjekte des Vertragsschlusses nur im Falle der privaten Konsoziationen nicht notwendiger Art die insoweit freien Individuen sind; auf allen anderen Stufen handelt es sich jedoch um soziale Einheiten. Daher ist Gierkes Diktum, Althusius sei der - noch dazu später nicht mehr übertroffene - Urheber der modernen Gesellschaftsvertragstheorie, nicht haltbar, weil der Individualismus für die moderne kontraktualistische Tradition konstitutiv ist.

- Weiterhin macht Althusius von der Figur des "stillschweigenden Vertrages“ Gebrauch, der aber die ihm angesonnene Begründungslast, nämlich die individuelle Verpflichtung aller Mitglieder der Konsoziation zu tragen, nicht gewachsen ist, da die individuelle Autonomie der Vertragschließenden schon auf der untersten Ebene der Politeumata nicht (mehr) gegeben ist ${ }^{16}$.

b) Die Substantialisierung der politischen Einheit

- Die Lektion, welche die Bartholomäusnacht für die reformierte Staatslehre hinsichtlich der Folgen der Zwei-Reiche-Lehre für die Zukunftschancen religiöser Minderheiten darstellte, hat Althusius zum Postulieren der Identität von Bürger- und Glaubensrolle veranlaßt - auch wenn die administratio des aus dem göttlichen Erlösungshandeln folgenden religiösen Auftrags bei ihm institutionell ausdifferenziert ist. Das führt nicht nur dazu, daß Althusius die Rechte der religiös Andersgläubigen, und sei dies die Mehrheit der Bürger, massiv beschneidet ${ }^{17}$; sondern es führt $\mathrm{z}$. B. auch in den reformierten Universitäten zu einer massiven Abwehr der Versuche, die religiöse Meinungsäußerungsfreiheit zu etablieren ${ }^{18}$. Der Folgelast der Entfaltung der auch auf öffentliche Äuße-

15 Vgl. zum Folgenden vor allem Kersting (wie Anm. 2), 222 ff.; ambivalenter in der Einschätzung: Wolfgang Krawietz, Kontraktualismus oder Konsozialismus? Grundlagen und Grenzen des Gemeinschaftsdenkens in der politischen Theorie des Johannes Althusius, in: Politische Theorie (wie Anm. 7), 391-423. Es gehört zu den Paradoxien der Wirkungsgeschichte von Theorien, daß das gegen diese an Althusius anschließende, ihn jedoch vielfach verfassungs- und legitimationstheoretisch unterbietende Tradition deutscher Staatsrechtslehre geschriebene Werk von Ferdinand Tönnies, Gemeinschaft und Gesellschaft: Grundbegriffe der Soziologie (Darmstadt ${ }^{8} 1979$ ), zum Ansatzpunkt einer politisch regressiven Renaissance des Gemeinschaftsdenkens in Deutschland dienen konnte. Zu Ansatz und Durchführung der Tönniesschen Konzeption von Soziologie, die auch und vor allem Staatssoziologie ist, vgl. Manfred Walther, Gemeinschaft und Gesellschaft bei Ferdinand Tönnies und in der Sozialphilosophie des 17. Jahrhunderts oder Von Althusius über Hobbes zu Spinoza und zurück, in: Hundert Jahre Gemeinschaft und Gesellschaft: Ferdinand Tönnies in der internationalen Diskussion, hrsg. von Lars Clausen, Carsten Schlüter (Opladen 1991) 83-106, zu Althusius bes. $87 \mathrm{f}$.

16 Vgl. dazu Kersting (wie Anm. 2), 31 f.

17 Vgl. Schmidt-Biggemann, in: Politische Theorie (wie Anm. 9), 231.

18 Das kann man besonders gut an den Reaktionen beobachten, welche Spinozas Tractatus 
rungsfreiheit dringenden religiösen Subjektivität im Gefolge der Reformation konnte, wie die Folgezeit - und wie selbst noch Hobbes' Überwindungsversuch - zeigen wird, diese Konzeption der Einheit von Glaubens- und Bürgergemeinde nicht standhalten.

- Zum anderen vermochte auch die forcierte Art und Weise, in der Althusius auf einer gemeinsamen, alle necessaria und utilia teilenden Lebensführung besteht, dem ökonomischen Expansionsdrang gegenüber einer allumfassenden sittlichen Bindung nicht standzuhalten, ohne ebenfalls zu regredieren. Die Invektiven gegen überhöhte Handelsgewinne (II/35.36), Luxusgüter, unwürdige Berufe und unehrliche Gewerbe (II/30.31), die durchaus zum Repertoire der traditionellen, von sittlicher Gemeinschaftsbildung ausgehenden mittelalterlichen Wirtschaftsethik gehören, sind dafür ein deutliches Indiz ${ }^{19}$.

Mit der Spaltung der Staatssouveränität schließlich, in die sich der korporativ gefaßte populus, die Ephoren und der Magistrat teilen, ist die Aufgabe der Behauptung der politischen Einheit auseinanderstrebender gesellschaftlicher Kräfte, wie sie bevorsteht, nicht zu bewältigen. Vielmehr ist hier eher die Grundlage für einen Bürgerkrieg gelegt ${ }^{20}$. Mit der Betonung des Umstandes, daß sich als verpflichtend verstandene Lebensregeln immer auch aus naturwüchsigen sozialen Prozessen und geteilten Lebenswelten herausbilden, hat Althusius freilich ein Problem bezeichnet, dessen Nichtbeachtung alle politischen Theorien um ihren Realitätsgehalt bringt ${ }^{21}$. Was die kommunalistische Erfahrung betrifft, so hat die

theologico-politicus auch bei den Reformierten, und auch in Herborn, hervorgerufen hat; die Kritik und Zurückweisung richtet sich nämlich zunächst - und überraschenderweise - weniger gegen die historisch-kritische Methode der Bibelauslegung, die Spinoza entwickelt, sondern ist auf das 20. Kapitel des TTP über die Meinungsfreiheit gerichtet. S. dazu Manfred Walther, Machina civilis oder von deutscher Freiheit: Formen, Inhalte und Trägerschichten der Reaktion auf den politiktheoretischen Gehalt von Spinozas „Tractatus theologico-politicus", in: Le Tractatus théologico-politicus de Spinoza et la réception imméditate du spinozisme (1670-1677), éd. Paolo Cristofolini (Amsterdam, Maarssen, 1995) (im Druck).

19 Vgl. dagegen eine Gesprächsäußerung Spinozas, die Lambert van Velthuysen überliefert hat: Unter den „errores" Spinozas führt er an „vitium \& peccatum tam accommodatum esse ad vitam civilem colendam, ad pacem conservandam, \& ad societatem felicem, \& omnibus divitiis affluentem reddendam quam virtutem“ - „daß Laster und Sünde in gleicher Weise geeignet sind, das bürgerliche Leben zu kultivieren, Frieden zu halten und die Gesellschaft glücklich und von Reichtümern überfließend zu machen wie die Tugend". Diese Mandevilles These von „private vice - public virtue" vorwegnehmende Äußerung Spinozas hat Wim Klever aus den Werken Velthuysens ausgegraben, s. Wim Klever, Verba et sententiae Spinozae or Lambert van Velthuysen (1622-1685) on Benedictus de Spinoza (Amsterdam, Maarssen, 1991) 40 .

${ }^{20}$ So Scbmidt-Biggemann, in: Politische Theorie (wie Anm. 9), 229. Die Herausforderung, welche die Existenz konfessionell gemischter $=$ gespaltener Bevölkerungen für die Luthersche Zwei-Reiche-Lehre darstellt, ist auch für die weitere politische Geschichte in Deutschland sorgfältig zu untersuchen.

${ }^{21}$ Darauf, als auf das „soziologische“ Entwicklungspotential der Althusianischen Theorie, weist Krareietz, in: Politische Theorie (wie Anm. 15), hin, und dieser Gesichtspunkt bildet zugleich einen Anknüpfungspunkt für Spinozas, sich gegen die neuzeitliche Kontraktualismustheorie stellende, politische Theorie: s. unter 5.

Um die Frage, wie solche gemeinschaftsgebundenen Dispositionen sich in der modernen li- 
Form, in der Althusius glaubte, an ihr festhalten zu können, sowohl wegen der Ausdünnung des Prinzips direkter Demokratie als auch wegen der Überbürdung der politischen Synthesis mit religiösen und sittlichen Anforderungen, seine „Politica" nicht lange überlebt.

\section{Thomas Hobbes oder Die staatsabsolutistische Logik der Souveränität}

Zwischen Althusius auf der einen, Hobbes und Spinoza auf der anderen Seite liegt nicht nur ein halbes Jahrhundert und mehr, sondern auch die Erfahrung weiterer religiöser Bürgerkriege. Darauf muß politische Theorie, die Relevanz für ihre Zeit beansprucht, reagieren. Die Reaktionsweisen von Hobbes und Spinoza stimmen darin überein, daß beide die Existenz einer obersten souveränen, d. h. ungeteilten, Staatsgewalt für alternativlos notwendig halten. Die Reaktionen beider unterscheiden sich aber zugleich: Während Hobbes auf eine staatsautoritäre Lösung setzt, bei der das kommunalistische Moment ganz verschwindet, sucht Spinoza die Lösung in einer prozeduralen Verflüssigung und konstitutionellen Bindung der Herrschaft, die unmittelbar demokratieaffin ist. Das ist, für Hobbes, weil bekannt, knapper, für Spinoza, weil seit 1933 weniger bekannt, ausführlicher darzulegen.

4.1 Thomas Hobbes (1588-1679) entwickelt seine politische Philosophie unter der Bedingung, daß das Vertrauen in eine vorgegebene Natur- und darauf gegründete Pflichtenordnung, sei sie offenbarungs- oder kosmos-religiöser Provenienz, nicht mehr vorhanden ist. Weder auf ein den Menschen vorgegebenes Naturrecht, etwa im Sinne der althusianischen Gleichsetzung von Dekalog und Naturrecht, noch auch auf eine objektive Naturteleologie alles Seienden kann er seine politische Philosophie mehr gründen. Ausgangspunkt seiner Theorie von Staat und Recht wie von Herrschaft ist also die - auf analytischem Wege aus der Gegenwart des Gesellschaftslebens gewonnene-ursprüngliche Beschaffenheit des Menschen inmitten einer gegen seine Selbsterhaltungsbedürfnisse gleichgültigen Umwelt. Das Beweisprogramm des Hobbes besteht darin, die gewillkürte Schaffung einer staatlich-institutionellen Herrschaftsordnung als die einzige Möglichkeit gesicherter Lebensführung für gleichzeitig nebeneinander existierende Menschen sowie einen bestimmten Inhalt dieser institutionellen Ordnung als Inbegriff der Realisierungsund Stabilitätsbedingungen friedlicher Koexistenz von Menschen nachzuweisen. Das Mittel, mit dem die Menschen der naturwüchsigen, ihre Existenz gefährdenden Form ihres Zusammenlebens, dem Naturzustand, entkommen können, in dem jeder selbst über die tauglichen Mittel der Selbsterhaltung entscheidet, also seine natürliche Handlungsfreiheit gebraucht - eine Willensfreiheit im Sinne der Nichtdeterminiertheit der Inhalte des eigenen Wollens bestreitet er -, ist der allseitige Austauschvertrag, der sich aufgrund seiner zukunftsbezogenen Dimension als reziprokes Versprechen zeigt: Alle zusammenlebenden Individuen tauschen wechselseitig eine Verzichterklärung auf Ausübung ihrer natürlichen Rechte zugunsten eines

beralistisch geprägten Welt erhalten oder restituieren lassen, kreist auch das Denken der Kommunitaristen (vgl. 7.2). 
nicht involvierten Dritten - oder mehrerer solcher Dritter - aus. Alle moralischen und rechtlichen Verbindlichkeiten, denen die Menschen im staatlichen Leben, also als Bürger, unterliegen, sind durch Selbstbindung erzeugte Pflichten. Das heißt, daß auch alle staatliche Herrschaft ihren Rechtsgrund in einem Akt der Selbstbindung der Herrschaftsunterworfenen hat, d. h. daß Herrschaft aus Selbstunterwerfung resultiert. Diese Selbstunterwerfung ist insofern eine freie, d. h. durch nichts Vorangehendes begründete Unterwerfung und Verpflichtung, als es keine präexistierende Normen- und Pflichtenordnung gibt; sie ist insofern freilich erzwungen, als sie sich für die instrumentelle Vernunft als die einzige Möglichkeit zeigt, wie die Menschen ihre als ontologische Qualität bestimmte, ihnen daher von Natur aus vorgezeichnete Selbsterhaltung effektiv ins Werk setzen können.

4.2 Hobbes geht nun so vor, daß er zunächst Verhaltensregeln entwickelt, die deshalb Anspruch auf universelle Geltung machen können, weil sie von der Vernunft als taugliche Mittel der Selbsterhaltung nachgewiesen werden können; das sind die lawes of nature als Inbegriff moralischer Gesetze, welche, indem sie die natürliche Freiheit der einzelnen auf die Bedingungen friedlicher Koexistenz einschränken, „im Gewissen verpflichten“. Kehren so die Inhalte der traditionellen Naturrechtsund Pflichtenlehre wieder, so scheint doch ihr Status gegenüber der Tradition radikal verändert: Sie sind nicht mehr unbedingt verpflichtende Normen, sondern hypothetische Imperative, die angeben, wie sich Menschen verhalten müssen, wenn sie friedlich und sicher koexistieren wollen. In einem zweiten Schritt wendet Hobbes sich dem Problem zu, das seit jeher die crux der Naturrechtslehren war, nämlich der Frage nach den Wirksamkeitsbedingungen jener Gesetze in der sozialen Interaktion. Da alle aus Erfahrung wissen, daß auf die spontane Vernunftleitung des Handelns bei anderen, aber auch bei einem selber, kein Verlaß ist, wäre es gerade irrational, sich gemäß den natural lawes zu verhalten, ohne daß ein zureichender Grund für die Erwartung bestünde, daß die anderen sich auch daran halten: damit zerstörte man gerade die eigene Existenz, statt sie zu sichern. Indem Hobbes nun das ZweckMittel-Schema noch einmal anwendet, wobei jetzt die Erwartungssicherheit bezüglich der Orientierung aller an den lawe of nature der Zweck ist, weist er nach, daß nur die Existenz einer mit dem Gewaltmonopol ausgestatteten Herrschaftsagentur, ein staatlicher Souverän, das gesuchte taugliche Mittel ist.

Folglich muß der zwischen allen zu schließende und alle wechselseitig verpflichtende Vertrag beides, sowohl die Geltung der natural lawes (1) als auch die Bedingungen ihrer Wirksamkeit (2), enthalten. Die Formel für den Gesellschaftsvertrag lautet:

"I authorize and give up my rigth of governing myself, to this man, or to this assembly of man, on this condition, that thou give up the rigth to him, and authorize

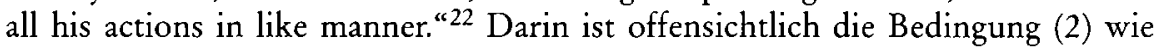
folgt erfüllt:

22 Thomas Hobbes, Leviathan: or the Matter, Form, and Power of a Commenwealth, Ecclesiastical and Civil = The English Works of Thomas Hobbes, vol. 3, ed. by William Molesworth (2nd repr. Aalen 1966) zit. nach Kapitel: Seite(n); 11: 158. 
- Indem zunächst alle sich wechselseitig verpflichten, auf die unbeschränkte Dispositionsbefugnis (das natural right) über die Tauglichkeit von Mitteln der Selbsterhaltung zu verzichten, ist der naturwüchsige Antagonismus einander gegenüber- und entgegenstehender je individueller Selbsterhaltungsmächte beseitigt.

- Indem dieser Verzicht nicht unbestimmt, sondern zugunsten bestimmter anderer Menschen erfolgt, ist zugleich gesichert, daß diese anderen eine ausreichende Macht der Durchsetzung der von ihnen für tauglich zur Selbsterhaltung aller gehaltenen Mittel haben; daß alle zugunsten Dritter verzichten, heißt nämlich, daß diese Dritten von den Kontrahierenden uno actu authorisiert werden, stellvertretend für jeden von ihnen zu handeln, daß sich also alle das Handeln der Dritten als ihr eigenes zurechnen lassen (müssen).

An die Stelle der je individuellen, miteinander um „Lebensmittel“ und um Macht konkurrierenden Individuen ist so ein Repräsentant (oder sind mehrere Repräsentaten) ihrer aller getreten, und nur in der Einheit der Repräsentanz kann eine Mehrheit von Individuen als Einheit agieren (L 16: 151). Und Hobbes spricht sich, unter dem Gesichtspunkt der Einfachheit und Durchschlagskraft, dafür aus, daß dieser Repräsentant des gemeinsamen Willens einer ist, ein Monarch als Souverän. Die so begründete Souveränität des Inhabers der Staatsgewalt ist jedoch nicht an bestimmte rechtliche Bedingungen gebunden, die ihm beim Vertragsschluß, im Sinne der traditionellen Herrschaftsverträge, abgenötigt oder auferlegt würden - vgl. etwa das Modell des Althusius für die Magistrate. Und das aus dem Grunde, daß bei einem Streit zwischen den Kontrahierenden und dem Souverän ja wiederum jener Antagonismus auftreten würde, den zu überwinden Zweck des gesamten Vertragsschlusses war. Dann fehlten dem Souverän nämlich die Mittel, welche seinem Gesetzesbefehl die Wirksamkeit garantieren. Deshalb ist der Vertrag auch so gefaßt, daß der Authorisierte oder die Authorisierten selber nicht Vertragspartner, sondern nur Begünstigte(r) des Gesellschaftsvertrages sind.

Ist so eine souveräne Staatsgewalt, legibus soluta, durch die bindende Selbstverpflichtung der Kontrahenten entstanden, so ergibt sich gleichwohl ein Problem: Zwischen der funktionalen Vorgabe für das authorisierte Handeln des Souveräns - nämlich den laws of nature auch Wirksamkeit gegenüber Widerstrebenden zu verleihen (Merkmal 2), - und der dem Souverän verliehenen absoluten Setzungskompetenz klafft eine Lücke. Hobbes versucht, dieser Lücke in einer ihm eigentümlichen Fassung des Widerstandsrechts Rechnung zu tragen:

a) Erstens weist er darauf hin, daß selbst eine Situation, in der der Souverän sich in Gesetzgebung und anderen Autoritätshandlungen nicht an die natural laws hält, die staatliche Existenz für die Bürger sehr viel erträglicher, weil weniger riskant macht als der Naturzustand. Denn jetzt droht die Gefahr nicht mehr von jedermann, sondern nur vom Souverän. Rational aber ist es, von zwei Übeln das kleinere zu wählen.

b) Zweitens bemüht Hobbes sich um den Nachweis, daß ,(t)he law of nature and civil law, contain each other, and are of equal extent" (L 26: 253). 
- Das civil law umfaßt das law of nature deshalb, weil letzteres erst durch rechtsverbindliche Anordnung Gesetz im vollen Sinne des Wortes ist; das impliziert übrigens, wie auch an der Auslegungslehre bei Hobbes deutlich wird, daß das law of nature als - ungeschriebener - Teil des civil law zu betrachten ist (L 26: 253).

- Und umgekehrt ist das civil law deshalb Teil des law of nature, weil die Verpflichtung zum Gehorsam gegenüber dem Souverän und folglich gegenüber dem civil law Inhalt des geschlossenen Vertrages ist und das Halten von Verträgen Bestandteil des law of nature ist. Anders gesagt: Die Geltung des natural law erstreckt sich auch auf alle vertraglich eingegangenen Verpflichtungen, umfaßt also auch die Authorisierung des Souveräns zur Gesetzgebung (L 26: 253254).

c) Drittens stellt Hobbes mehrfach heraus, daß der Verzicht auf die Ausübung des natürlichen Rechts eines jeden im Authorisierungsvertrag zwar ein absoluter, aber kein unbeschränkter Verzicht ist. Die inhaltliche Beschränkung ergibt sich analytisch aus dem Zweck des Vertrages, nämlich ein sicheres und friedliches Leben zu garantieren. Nun kann aber meine Tötung durch den Souverän kein taugliches Mittel zu meiner Selbsterhaltung sein. Also kann die Aufgabe des Rechts auf Leben auch kein Bestandteil des Authorisierungsvertrages gewesen sein. Hier zeigt sich bei Hobbes eine Grundlage für die Konzeption unübertragbarer, unverzichtbarer Menschenrechte, und wenn man die verschiedenen Formulierungen des unantastbaren Kerns natürlicher Rechte, die sich bei Hobbes finden, im einzelnen durchgeht - am extensivsten sind sie beschrieben als „right to govern their own bodies; enjoy air, water, motion, ways to go from place; and all things else without which a man cannot live, or not live well (!) “ (L 15: 141) -, dann zeigt sich, daß die unaufgebbaren natürlichen Rechte, die den Widerstand der Bürger gegen den Souverän legitimieren, weit über das bloße Notwehrrecht zur Lebenserhaltung hinausgehen. Hobbes ist eben alles andere als ein totalitärer Denker, er ist ein - freilich autoritärer - Liberaler ${ }^{23}$.

d) Viertens schließlich ergibt sich aus dem zuletzt Gesagten, daß es auch ein Klugheitsgebot für den Souverän ist, seine Souveränität so auszuüben, daß die Bürger ihre natural rights, die sie behalten haben, nicht gefährdet sehen. Sonst geht er nämlich der Durchsetzungsmacht für seine Befehle und damit auch der Souveränität selber verlustig. Hier berühren sich also, so scheint es, Recht und Macht nicht nur, sondern es zeigt sich, daß das Recht des Souveräns die Macht zumindest zu ihrer Bedingung hat, wenn nicht gar Recht letztlich auch auf Macht reduziert ist.

4.3 Überblickt man die Hobbessche rationale Konstruktion des Staates in ihrem Verlauf und in ihrem Ergebnis, so sind, am Leitfaden des Kommunalismusproblems und im Vorgriff auf die nächste zu behandelnde Theoriegestalt, folgende kritischen Bemerkungen zu machen:

23 Vgl. dazu auch Den Uyl, Warner, Liberalism and Hobbes and Spinoza, in: Studia Spinozana 3 (1987) 319-347. 
a) Das natural right eines jeden, sich selbst mit den verfügbaren Mitteln so gut wie möglich zu erhalten, ist zugleich eine, vielmehr: die erste und einzige natürliche Pflicht. Denn die als geeignetes Mittel der Selbsterhaltung nachgewiesenen natural lawes gewinnen zwar ihren Verbindlichkeitscharakter letztlich aus der Selbstverpflichtung, diese Selbstverpflichtung ist aber bei Hobbes zugleich eine Pflicht: „A law of nature, lex naturalis, is a precept or general rule, found out be reason, by which a man is forbidden to do that, which is destructive of his life, or taketh away the means of preserving the same" (L 14: 116-117; meine Hervorhebung. Wa.). Das heißt nämlich, daß die menschliche Vernunft zwar die Entdeckung des Inhalts aller natürlichen Gesetze leistet, daß diese Regeln aber, als Vorschriften über das, was zu tun und zu unterlassen ist, aufgrund ihrer alternativlosen Tauglichkeit zur Selbsterhaltung verbindlich sind. So bewahrt Hobbes immer noch, wenn auch in einer letzten Schwundstufe, den Kontakt zum normativen Naturrechtsdenken der Tradition.

b) Einen Vertrag zu halten, kraft dessen ich eventuell als erster zu leisten habe, bevor sicher ist, daß die anderen das auch tun, ist, wie Hobbes selber einschärft, unvernünftig. Nun weiß ich aber zum Zeitpunkt des Abschlusses des Vertrages nicht, ob nicht die anderen, wenn sie gemäß dieser Selbstverpflichtung später das Ihre leisten müssen, dies auch tun; eventuell bin ich aber der erste, der erfüllen muß. Also ist es unvernünftig, als erster gemäß dem eingegangenen Vertrage zu leisten. Damit ist aber unsicher, ob derjenige, dessen Befehl ich mich unterwerfe, wirklich der Souverän ist; und diese Unsicherheit hat ein jeder in Bezug auf alle anderen; Hobbes hat dem, scharfsinnig wie er ist, auch durchaus Rechnung getragen, freilich ohne das besonders kenntlich zu machen. So schreibt er an derjenigen Stelle, an der das Problem auftritt, nämlich bei Behandlung der Frage, wie denn die Einigung auf den oder die Begünstigten zustande kommt:

"Thirdly, because the major part hath by consenting voices declared a sovereign; he that dissented must now consent with the rest; that is, be contented to avow all the actions he shall do, or else justly be destroyed by the rest" (L 18: 162; meine Hervorhebung. Wa.).

Was zunächst einfach als die Hobbessche Form der Begründung klingt, mit der auch schon zuvor die Verbindlichkeit von Mehrheitsentscheidungen für die Überstimmten gerechtfertigt wurde, erweist sich bei genauer Betrachtung als der alles andere als kontraktualistische Modus, in dem Herrschaft überhaupt zustande kommt: Jeder einzelne sieht sich nämlich jetzt, als Mitglied einer zu gemeinsamer Willensbildung zusammengekommenen Gruppe, jeweils dem „Rest“ gegenüber, er befindet sich in einer asymmetrischen Gruppensituation. Denn für jeden sind die anderen jeweils dieser Rest, und zugleich gehört jeder aus der Sicht jedes anderen zu diesem Rest. Und es ist eben diese allseitige Antizipation der anderen als Rest, dem gegenüber sich jeder als machtunterlegen weiß, was ihm die Zustimmung abnötigt. Eben durch die wechselseitige Unterstellung aller anderen als des machtüberlegenen Restes konstituiert sich also der Souverän. Es handelt sich um eine sich selbst realisierende Fiktion. Damit allererst ist der Zirkel der kontraktua- 
listischen Begründung der Souveränität vermieden, der darin besteht, daß eine Rechtspflicht zum Verzicht auf eigenmächtiges Handeln nur gegenüber einer schon vorhandenen souveränen Gewalt besteht, die doch andererseits erst aus der vertraglichen Selbstverpflichtung hervorgehen soll. Damit ist aber die kontraktualistische Begründung der Souveränität verlassen ${ }^{24}$.

c) Der Kontraktualismus schließt zwar insofern an die kommunalistische Erfahrung an, als er die Legitimität von Herrschaft nicht mehr transzendent, sondern ausschließlich aus dem expliziten Konsens der Vertragspartner begründet, also die - ihrer Logik nach demokratische - Konstitution des Staates durch die Kontrahierenden lehrt. Jedoch ist, wie ausführlich dargestellt, das Resultat aufgrund der Lücke zwischen der moralphilosophischen Begründung und der absoluten Setzungskompetenz des Souveräns von zweifelhaftem Wert, dient der Vertrag doch dazu, die Vertragspartner kraft eigenen Willens irreversibel in die Position des bloßen Untertanen herabzusetzen. Otto von Gierke hat das die „naturrechtliche Vernichtung des Naturrechts" genannt.

d) Schließlich ist das, was Hobbes über das Auslaufen der Verpflichtung gegenüber dem Souverän sagt, wenn er faktisch die Macht verliert, auf seine Implikationen hinsichtlich des Verhältnisses von Recht und Macht bei Hobbes und in der Sache zu bedenken.

e) Ein Gesichtspunkt, den ich in der Hobbes-Darstellung nicht berührt habe ${ }^{25}$, ist abschließend zu bedenken: das Verhältnis von Staatsauthorität und Religion, das theologisch-politische Problem: Hobbes läßt den Souverän den großen Definierer wie aller öffentlich äußerungsfähigen, so auch der religiösen Wahrheit sein. Zwar läßt er das Innere des Menschen als staatsfreien Raum übrig - was Carl Schmitt dann später als aus Agnostizismus von Hobbes kozediertes und von Spinoza konsequent genutztes Einfallstor der subjektivistischen Unterhöhlung der Staatsautorität brandmarken wird ${ }^{26}$-, aber die Unterdrückung jeder öffentlichen Äußerung nicht staatlich autorisierter religiöser Überzeugung tut der in der Reformation aufgebrochenen religiösen Subjektivität in einem Maße Gewalt an, das sich nur destabilisierend, desintegrativ auf die politische Einheit der Gesellschaft auswirken kann.

\footnotetext{
${ }^{24}$ Ich folge hier der Analyse von Osamo Ueno, Spinoza et le paradoxe du contrat social de Hobbes: „Le reste“, in: Cahiers Spinoza 6 (printemps 1991) 269-295.

${ }^{25}$ Vgl. dazu ausführlich Manfred Walther, Biblische Hermeneutik und/oder theologische Politik bei Hobbes und Spinoza, in: Hobbes e Spinoza: Scienza e politica, ed. Daniela Bostrenghi (Napoli 1992) 623-669.

26 „Schon wenige Jahre nach dem Erscheinen des, Leviathan ${ }^{\circ}$ ficl der Blick des ersten liberalen Juden auf die kaum sichtbare Bruchstellc": Carl Schmitt, Der Leviathan in der Staatslehre des Thomas Hobbes: Sinn und Fehischlag cines politischen Symbols (Köln 1982; zuerst 1938) 86.
} 


\section{Benedikt de Spinoza oder Die Macht der Demokratie}

5.1 Es kennzeichnet den Rang Spinozas sowohl als eines der scharfsinnigsten Hobbes-Leser in der Wirkungsgeschichte der Hobbesschen politischen Theorie als auch als eines systematischen Denkers, daß er just jene soeben angemerkten Brüche und Lücken erkennt und in einer Hobbes radikalisierenden und gerade dadurch dessen Lösung sprengenden Denkbewegung eine neue Gestalt der politischen Theorie hervorbringt, die wegen eben dieser Radikalität des Ansatzes wie des eingeschlagenen Lösungsweges lange Zeit - sieht man einmal von den Schotten $\mathrm{ab}^{27}$ - für die allgemeineuropäische politische Philosophie nicht anschlußfähig sein sollte:

Er beseitigt nämlich rigoros den letzten normativ-naturrechtlichen Rest an der Basis der Hobbesschen Rechts- und Staatstheorie (ad 4.4a), verwirft, jedenfalls in der reifen Gestalt seiner Theorie im unvollendet hinterlassenen Tractatus politicus, die kontraktualistische Staatstheorie (ad 4.4b) und beseitigt die Lücke zwischen dem gemeinsamen Willen der Vielen und der Staatsgewalt durch Prozeduralisierung des Verhältnisses zwischen beiden und durch das Einfügen konstitutioneller Momente (ad 4.4c), faßt Recht konsequent als Macht (ad 4.4.d) und begründet schließlich das Recht öffentlicher Meinungsäußerung in religiösen und wissenschaftlichen, aber auch in politischen Angelegenheiten ( $\mathrm{ad} 4.4 \mathrm{e})$ - und wird damit zum Begründer einer normativ entlasteten, dafür aber umso realitätsnäheren Theorie der Demokratie als der natürlichsten Regierungsform und beeinflußt auch jene Form der modernen Demokratietheorie bedeutsam, die uns von Rousseau bekannt ist.

5.2 Spinoza versucht nachzuweisen, daß subjektive Vernunft und Freiheit keine ursprünglichen (im Sinne von anfänglichen) Gegebenheiten menschlicher Existenz sind, sondern sich allererst im Zuge der Gesellschafts- und Staatsbildung herausbilden und daß die Demokratie diejenige Regierungsform ist, in der die Handlungsmacht der Gesamtgesellschaft und eine gesicherte Sphäre der Entfaltung und Betätigung des „Eigensinnes“ der Bürger, also feste subjektive Rechte der Individuen, sich im größtmöglichen Maße wechselseitig stabilisieren und die, weil sie die besten Entfaltungschancen für die dem Menschen innewohnenden Möglichkeiten bietet, insofern zugleich die natürlichste aller Regierungsformen ist.

Das Ignorieren und Leugnen der Tatsache, daß die Menschen zunächst und zumeist, aufgrund des vielfältigen Einwirkens der Umweltdinge auf sie, in ihren Präferenzen und Handlungen mehr durch die "blinde“ Macht der Leidenschaften, also durch Affekte, bestimmt sind als durch vernünftige Deliberation, ist für die politischen Theorien der „Philosophen“ kennzeichnend: Indem diese die Affektbestimmtheit menschlichen Handelns nicht als deren Natureigenschaft, sondern als verdammungswürdige Fehler qualifizieren, „glauben (sie) dergestalt etwas Erhabenes zu tun und den Gipfel der Weisheit zu erreichen". Dem liegt ein realitäts-

$27 \mathrm{Vgl}$. Wim Klever, Hume contra Spinoza? in: Hume Studies 16 (1990) 89-105; ders., More about Hume's Debt to Spinoza, in: Hume Studies 19 (1993) 55-74. 
fernes Menschenbild zugrunde: „Sie stellen sich freilich die Menschen nicht vor, wie sie sind, sondern wie sie sie haben möchten“, und haben daher „statt einer Ethik meistens eine Satire geschrieben", ihre Theorien haben den Charakter von Utopien $^{28}$. Folgt man jedoch der Einsicht in die grundlegende Affektbestimmtheit menschlichen Lebens, so hat das zur Folge, daß auch das natürliche Recht der Menschen, d. h. das Recht in ihrem ursprünglichen Zustand, durch das Wirken der allgemeinen Naturgesetze menschlichen Affektlebens und nicht durch moralische, gar vernunftgenerierte, Normen bestimmt ist.

Hatte Hobbes das natürliche Recht eines jeden definiert als die Freiheit der Realisierung eigener Handlungsentwürfe aufgrund seiner eigenen Macht ohne äußere Hindernisse, so zeigt Spinoza, daß diese Freiheit und damit auch jenes natürliche Recht praktisch extensionslos sind. Denn das Aufeinandertreffen von Menschen, die so von ihren blinden Begierden bestimmt sind, produziert einen $\mathrm{Zu}$ stand, in dem niemand etwas als dauerhaft seines behaupten kann, so wie Hobbes es mit der Formel vom „Krieg aller gegen alle“ herausgestellt hatte: „Das natürliche Recht des Menschen, solange es durch die Macht eines einzelnen bestimmt wird und dieser ein auf sich allein gestellter einzelner ist, (ist) folglich so gut wie nichts...; es besteht eher in der Einbildung als in der Wirklichkeit, fehlt doch jede Sicherheit, seiner inne zu sein" (TP 2/15). Das ursprüngliche Naturrecht eines jeden ist also, wie Spinoza, Hobbes konsequent zu Ende denkend, ausführt, praktisch eine leere Menge. Das aber heißt, daß der Naturzustand ein anomischer Zustand - im Sinne normativer Gesetze - ist, daß die Menschen in ihm „ebensowenig verpflichtet (sind), nach den Gesetzen der gesunden Vernunft zu leben, als die Katzen verpflichtet sind, nach den Gesetzen der Löwennatur zu leben “29. „Unter eigenem Recht" steht ein Mensch jedoch nur insoweit, "wie er alle Gewalttaten zurückweisen und einen ihm zugefügten Schaden nach eigenem Gutdünken vergelten kann, d. h., uneingeschränkt formuliert, wie er nach seiner eigenen Sinnesart leben kann" (TP 2/9); und über die dazu erforderlichen Mittel verfügt der von ursprünglichen, d. h. rein naturwüchsigen, Affekten bestimmte Mensch des Naturzustandes eben nicht.

Daraus folgt, daß von tatsächlich gegebenen subjektiven Rechten der Menschen nur da die Rede sein kann, wo es zu Kooperationen kommt: „Wenn zwei auf einmal zusammenkommen" - Spinoza meint das als "auf natürliche Weise zusammenkommen", "naturaliter convenire" (TP), formuliert also in bewußt nichtkontraktualistischer Terminologie - „und ihre Kräfte verbinden, dann vermögen sie zusammen mehr und haben folglich mehr Recht auf die Natur als jeder für sich allein. Und je mehr ihre Bedürfnisse so miteinander verknüpfen, desto mehr Recht haben sie alle zugleich" (TP 2/13; meine Übersetzung. Wa.). Daraus wiederum folgt, „daß ein Recht der Natur, das dem Menschengeschlecht eigen ist,

28 Baruch de Spinoza, Politischer Traktat / Tractatus politicus (Hamburg 1994) zit. nach Kapitel/Paragraph: $1 / 1$.

29 TTP 16: 233. Der Vergleich spricht zugleich aus, daß der in seinem Handeln von Vernunft bestimmte Mensch mächtiger ist als der seinen Leidenschaften ausgelieferte. 
kaum begriffen werden kann, als wo die Menschen gemeinsame Rechte (iura communia) haben" (TP 2/15; eigene Übers.). Alles gehaltvolle Menschenrecht ist also gesellschaftlich, durch Kooperation, konstituiertes Recht, ist ius commune - mit der Folge, daß es für den Menschen, sofern er als in sozialen Beziehungen stehend genommen wird, kein ursprünglich eigenes Recht gibt, daß alles dem einzelnen zukommende Recht vielmehr nur „dasjenige (ist), das das gemeinsame Recht ihm zugesteht" (TP 2/16) ${ }^{30}$. Damit ist nachgewiesen, daß alles für den Menschen charakteristische, d. h. ihn von anderen Bestandteilen der Wirklichkeit unterscheidende Recht nicht naturgegeben ist, sondern kulturell hervorgebracht ist - wenn auch die Bedingungen seiner Wirksamkeit immer die Naturgesetze menschlicher Präferenzbildung und Handlung bleiben.

5.3 Die Möglichkeit, die Entstehung eines weitgehend gegenüber den Interventionen der Mitmenschen gesicherten Rechts als aus rationalem Selbsterhaltungskalkül kontraktualistisch - zumindest indirekt - produziertes Recht zu erklären und damit die solche Rechte gewährenden Herrschaftsverhältnisse rational zu legitimieren, hat sich Spinoza dadurch abgeschnitten, daß er die dazu erforderliche wissenschaftlich aufgeklärte instrumentelle Vernunft nicht zu den Naturgegebenheiten menschlicher Existenz zählt, solche subjektive Vernünftigkeit vielmehr als allererst im Prozeß der Vergesellschaftung sich herausbildend begreift ${ }^{31}$. Auch die Gesetze, die nach Hobbes aus solch interessiertem Vernunftgebrauch resultieren, haben für Spinoza (noch) keinen Adressaten, sind also nicht als "natürlich“ qualifizierbar. Vielmehr verlangt Spinozas Beweisprogramm, daß er eine Theorie der naturwüchsigen Gesellschafts- und Staatsgenese liefert, die nur das voraussetzen kann, was ursprünglich dem Menschen gegeben ist, und das sind dessen Affekte.

Gesellschafts- und Staatsbildung ergeben sich für den Menschen, der sich als ohnmächtiger in eine nicht auf seine Lebensbedürfnisse teleologisch zugeschnittene ${ }^{32}$, sondern gegen diese gleichgültige Umwelt „geworfen" sieht und der doch ontologisch durch das Selbsterhaltungsstreben (conatus in suo esse perseverandi) charakterisiert ist, kraft der Notwendigkeit seiner Natur vielmehr aus der zunächst und zumeist naturwüchsigen Interaktion affektbestimmt agierender Menschen. Die Pointe dieser Theorie ist, daß der Mensch „immer schon“ in Sozialbeziehungen lebt, weil er ohne solche nicht zu leben vermag. Spinoza nutzt also das Theorem des Naturzustandes, um diesen selber und das in ihm angeblich geltende

\footnotetext{
30 Als schlagendsten Beleg dafür, daß die subjektiven Rechte der Bürger gesellschaftlich konstituierte und garantierte Rechte sind, führt Spinoza das Erbrecht an, dessen Träger, im üblichen Verstande, ja schon „untergegangen“ ist, wenn es wirksam wird: TP 7/25.

31 Vgl. zu diesem Spezialproblem Manfred Walther, Die gesellschaftliche Begründung der Vernunft bei Spinoza, in: Truth, Knowledge and Reality: Inquiries, in: The Foundation of Seventeenth Century Rationalism (Wiesbaden 1981) 44-55.

32 Vgl. die Destruktion teleologischer Weltdeutungen in Baruch de Spinoza, Ethik nach geometrischer Methode dargestellt (Hamburg 1976 u.ö.) Teil I, Anhang = 39-48. Zitiert wird die Ethik im folgenden nach Teil (1, 2, etc.) und Lehrsatz (L1, 2 etc.), also z. B. E 2L17 = Ethik, Teil I, Lehrsatz 17.
} 
Naturrecht als rationalistische, die Vermitteltheit durch Gesellschaft und Staat sich selber verdeckende Abstraktion aus den tatsächlich gegebenen sozialen Verhältnissen nachzuweisen.

Der Zwang zur Vergesellschaftung ergibt sich für ihn wie schon für die Sophistik und im Anschluß daran für Aristoteles und die Aristotelesrezeption zum einen daraus, daß der Mensch sich ohne das Eingehen von Kooperationsbeziehungen nicht erhalten kann. Solche Kooperation aber entsteht nicht aus zweckrationalem Kalkül, auch wenn die Fähigkeit zum Registrieren der Vorteile von Kooperation im Gedächnis bleiben und daraus sich entwickelnde Veränderungen der Präferenzen langfristig in Rechnung gestellt werden können, sondern aus der Mechanik der Affekte, wie Spinoza sie im 3. Teil seiner „Ethik“ vorgeführt hat ${ }^{33}$.

Diesem Zwang zur Kooperation korrespondiert in der Affektnatur des Menschen nun zum anderen ein Phänomen, das Spinoza imitatio affectuum nennt und das in der vorausliegenden Tradition als Mimesis und seit Hume als sympathy, später dann als Empathie bezeichnet wird. Es besteht darin, daß die Menschen dazu neigen, spontan Affekte, die sie bei anderen ihnen ähnlichen Dingen wahrnehmen, nachahmen, $d . h$. daß sich deren von uns wahrgenommene Affekte - sofern wir bezüglich dieser anderen noch keine Affekte gebildet haben - auf uns selber übertragen (E 3L27). „Mitleid“ - die Nachahmung der bei einem anderen wahrgenommenen Trauer - „und Ruhmsucht" - ein Affekt, der sich einstellt, wenn ich wahrnehme, daß ein anderer aufgrund einer mir zugeschriebenen Handlung sich freut (E 3L30) - „liegen der Geselligkeit [sociabilite], Herrschsucht und Neid liegen der Ungeselligkeit [àsociabilite] zugrunde, und diese beiden Gruppen von Leidenschaften sind untrennbar".

Sehen wir jemand, mit dem wir Mitleid empfinden, durch einen anderen verletzt, so stellt sich Empörung (indignatio) als spezifische Form des Hasses ein, und dies ist ein zu leidenschaftlicher Solidarisierung determinierender Effekt, der die reine Selbstzentriertheit des Handelns intersubjektiv aufsprengt und der, wie Spinoza ausführt, allen Revolutionen zugrunde liegt (TP 6/1). Diese Lehre Spinozas besagt nichts anderes, als daß es die Ichzentriertheit aufbrechend Affekte gibt, denen wir ausgesetzt sind und die in ihrem Zusammenspiel miteinander und mit den gegenläufigen Affekten die Herausbildung zunächst lockerer und kurzfristig bestehender, sodann längerfristiger und institutionell sich verfestigender sozialer Gruppen hervorbringen ${ }^{34}$.

${ }^{33}$ Ich folge in diesem Abschnitt der Interpretation, die Alexandre Matheron, Le problème de l'évolution de Spinoza du Traité théologico-politique au Traité politique, in: Spinoza: Issues and directions (wie Anm. 4), 258-270, vorgelegt hat, wo dic antikontraktualistische Struktur des TP mit großer Klarheit herausgearbeitet wird.

${ }^{34} \mathrm{Vgl}$. dazu im Detail Matheron 1990 (wie Anm. 32), 263 ff. Mit der kontraktualistischen Staatsbildungstheorie ist somit auch jede ohne Rekurs auf die Affekte oder Emotionen arbeitende Form rationaler Handlungstheorien, wie sie in den verschiedenen Theorien des rational choice vorliegen, schon im Ansatz kritisiert - obgleich sich Spinoza auch dieser Theoriefigur ausgiebig bedient! Der zweckrationale Kontrakt läßt sich dann als eine unter bestimmten Bedingungen mögliche Spezialform der affektiv bestimmten Interaktion interpretieren. 
Kehren wir zu den Konstitutionsbedingungen subjektiver Rechte zurück, so ergibt sich aus der Tatsache, daß inhaltlich gehaltvolle Rechte immer aufgrund von Kooperation entstehen, also iura communia sind, „daß das Recht des Staates oder der höchsten Gewalt nichts anderes ist als eben das Recht der Natur, das durch die Macht, nun nicht mehr jedes einzelnen, sondern der wie von einem Geist geleisteten Menge bestimmt wird" (TP 3/2). Daß ein einzelner oder auch mehrere die Staatsgewalt ausüben können, heißt also nichts anderes, als daß die anderen, die damit als Untertanen qualifiziert sind, den Inhabern der Souveränität die Dispositionsgewalt über die Art der Ausübung ihrer je eigenen Handlungsmacht überlassen - und zwar nur solange, wie sie dazu aufgrund ihrer jeweiligen Affektlage disponiert sind, und es ergibt sich weiter, daß ,jeder Bürger zu Recht (tut oder besitzt) nur dasjenige, was er kraft eines gemeinsamen Beschlusses in Anspruch nehmen kann" (TP 3/2). Die Bereitschaft, anderen solche Dispositionsmacht einzuräumen, also Herrschaft zu akzeptieren, ergibt sich daraus, daß bei entsprechender Ausübung durch den oder die Herrscher die Gründe zur Furcht sich verringern und die Anlässe für Hoffnung sich vermehren, insbesondere wenn die durch diese Akkumulation von Handlungsmacht mögliche Rückverteilung des Rechts der Souveränität an die Bürger in Form subjektiver Rechte diesen einen - von der Macht der Gesellschaft, also vom ius commune institutionalisierten und garantierten - Raum für die Führung eines Lebens nach dem eigenen ingenium eines jeden ermöglicht. Denn dadurch wird wiederum die Bereitschaft der Bürger erhöht, solche Herrschaft zu akzeptieren, was wiederum vermehrte Möglichkeit der Einräumung bietet, etc.: Es bildet sich ein sich selbst verstärkender Machtkreislauf zwischen Bürgern und Souverän heraus, dessen Grundlage die Breite und Tiefe von Kooperationsbeziehungen ist.

5.4 Daraus, daß „das Recht der Regierung oder der höchsten Gewalt nichts anderes ist als eben das Recht der Natur, das durch die Macht, nun nicht mehr jedes einzelnen, sondern der wie von einem Geist geleiteten Menge bestimmt wird“ (TP 3/2), leitet Spinoza nun eine Reihe von Folgerungen ab:

a) Alle Herrschaftsmacht, also jede summa potestas, beruht auf der Akzeptanz durch die Beherrschten ${ }^{35}$. Die Macht und folglich das Recht des Souveräns, über die Handlungsmacht der Bürger zu disponieren, besteht also nur solange, wie sich beides durch den nicht stillbaren Machtkreislauf hierdurch reproduziert ${ }^{36}$. Denn niemand verzichtet je endgültig auf sein natürliches Recht, die Staatsleistungen und -institutionen gemäß der eigenen Präferenzstruktur zu beurteilen und gemäß den dieses Urteil begleitenden Affekten zu handeln.

35 „Oboedientia facit imperantem“, so hat Hugo Preuß, Vom Obrigkeitsstaat zum Volksstaat, in: Handbuch der Politik, 3. Bd. (Berlin; Leipzig ${ }^{3}$ 1921) 16-26 (16), diese „Weisheit Spinozas" bündig formuliert.

36 Das ist das von Ernst Renan, einem Spinoza-Forscher, formulierte "prébiscite de tous les jours". 
b) Diejenige Regierungs- oder Herrschaftsform ist daher die stabilste, in der jener Machtkreislauf, in welchem allein sich alle staatliche Herrschaft vollzieht, auch zum expliziten Operationsmodus des politischen Systems gemacht wird, d. h. die Demokratie. Denn in dieser „überträgt niemand sein Recht derart auf einen anderen, daß er selbst fortan nicht mehr zu Rate gezogen wird; vielmehr überträgt er es auf die Mehrheit der gesamten Gesellschaft, von der er selbst ein Teil ist. Auf diese Weise bleiben alle gleich, wie sie es vorher im Naturzustand waren "37.

c) Von der Wirksamkeit dieses „Naturgesetzes“ (vgl. TTP 16: 235) sind auch die Vertragshandlungen der Bürger nicht ausgenommen: Sie haben ihre Stabilität nur dadurch, daß sie Erwartungssicherheit begründen, und sei es, weil vom Vertragsbruch mehr Nachteile als Vorteile zu gewärtigen sind (TTP 16: 235-236). Das aber bedeutet, daß der Rechtssatz "pacta sunt servanda“ kein naturrechtlich, sondern nur ein positiv-rechtlich begründeter Satz ist, daß also die Erklärung der Staatsentstehung mit kontraktualistischen Argumenten eine petitio principii darstellt.

d) Der „Innenraum“ der Subjektivität ist zwar für den Inhaber der Staatsgewalt vielfältig manipulierbar, aber doch niemals ganz beherrschbar. Und je mehr die Menschen lernen, autonom und rational zu urteilen, umso weniger ist das der Fall. Wo aber keine Macht ist, ist auch kein Recht. Deshalb ist es nicht herrschafts- und ordnungsstabilisierend, sondern wirkt sich im Gegenteil destabilisierend aus, wenn die Staatsgewalt es untersagt, die eigenen Meinungen religiösen, wissenschaftlichen oder politischen Charakters öffentlich kundzutun. Denn wes der Sinn voll ist, des geht der Mund über. So begründet Spinoza es als Zeichen der Klugheit der Herrschaft in eigenem Interesse, öffentliche Redefreiheit zu gewähren, die auch Gesetzeskritik einbezieht, wird doch damit die Akzeptanz einer Regierung durch die Bürger erhöht ${ }^{38}$. Damit ist zugleich die bürgerliche Öffentlichkeit grundlegend begründet, und die Redefreiheit ist zugleich die erste Gestalt, in der durch politische und rechtliche Institutionen die naturrechtlich zunächst nur innere Freiheit einen äußeren Betätigungsraum erhält, der freilich, indem er Herrschaft stabilisiert, diese zugleich auch rechtlich beschränkt. Und es ist das Grundmuster der spinozanischen politischen Theorie sichtbar geworden, denn nach Spinoza ist „der Zweck des Staates... in Wahrheit die Freiheit" (TTP 20: 301).

e) Menschen, die die Konstitutionslogik von Staat und Recht reflexiv-subjektiv eingeholt haben und die wissen, „daß das Schwert des Königs, d. h. sein Recht, in Wahrheit der Wille der Menge selbst oder ihres einflußreichsten Teils“ ist, und die „als vernunftbegabte Menschen sich niemals ihres Rechts so begeben, daß sie aufhören, Menschen zu sein“, werden deshalb der Ausübung „ihrer" eigenen akkumulierten Handlungsmacht durch den Souverän konstitutionelle Schranken auferlegen. „Denn die Grundlagen des Staates sind als gleichsam ewrige Beschlüsse

37 TTP 16: 240 (meine Hervorhebung. Wa.). Da die Demokratiekapitel des TP von Spinoza nicht mehr fertiggestellt worden sind, greife ich hier auf die entsprechende Stelle des TTP zurück.

${ }^{38}$ Das ist ausführlich entwickelt in TTP 20! 
des Königs anzusehen, so daß seine Staatsdiener durchaus gehorsam sind, wenn sie sich weigern, seine Anordnungen auszuführen, sobald er etwas befiehlt, was sich mit den Grundlagen des Staates nicht verträgt ${ }^{\text {“39. }}$.

f) Die Macht und folglich das Recht des Inhabers oder der Inhaber der summa potestas, über die Handlungsmacht der Bürger zu disponieren, besteht nur solange, wie sich beides durch den niemals stillstehbaren Machtkreislauf hindurch reproduziert (s. o. unter a). Denn niemand verzichtet je endgültig auf sein natürliches Recht, die Staatsleistungen und -institutionen gemäß der je eigenen Präferenzstruktur zu beurteilen und gemäß den dieses Urteil begleitenden Affekten zu handeln. Damit ist auch eine unüberschreitbare Grenze des ansonsten von Spinoza als legibus soluta gekennzeichneten Souveränitätsrechts bezeichnet. „Denn es liegt sicherlich in der Natur des Menschen, sich zusammenzutun, sei es aufgrund einer gemeinsamen Furcht, sei es aus dem Verlangen, einen gemeinsam erlittenen Schaden zu rächen. Und weil das Recht des Gemeinwesens durch die gemeinsame Macht der Menge definiert wird, verringert sich sicherlich dessen Recht, d. h. dessen Macht, in dem Maße, in dem es selber Veranlassung gibt, daß sich viele zusammentun" (TP 3/9), d. h. daß eine Empörung entsteht ${ }^{40}$. Steht also eine Empörung niemals in Übereinstimmung mit dem bürgerlichen Recht, ist also jeder Widerstand illegitim, so bedroht doch eine solche Empörung, wenn sie erfolgreich ist, auch nicht den Bestand eines Gemeinwesens, sondern ,sie bewirkt nur, daß sie (d.i. die Bürger. Wa.) seine (d.i. des Gemeinwesens. Wa.) Form in eine andere umwandeln, dann nämlich, wenn sie ihre Streitigkeiten nicht unter Beibehaltung der Gestalt des Gemeinwesens beilegen können" (TP 6/2).

5.5 Auf der Grundlage der von allen normativen Bestandteilen gereinigten Bestimmung des "natürlichen", d. h. ursprünglichen Status des Menschen in der Welt als einer spezifisch verfaßten Selbsterhaltungs- und Handlungsmacht entwirft Spinoza eine Theorie der Gesellschafts- und Staatsbildung und -transformation, welche die von Menschen aufgrund des Zusammenspiels von Affekt und sich entwickelnder Vernunft produzierten rechtlichen und politischen Institutionen als Resultate eines nicht stillbaren Prozesses der Bildung und des Verfalls von Machtakkumulation begreift, der trotz der Rationalisierung, deren er fähig ist, weiterhin nur als Spezialfall, als spezifische kulturelle Konstellation, einer das Empfinden und das Denken wie das Handeln der Menschen bestimmenden Gesetzmäßigkeit begriffen wird. Gerade dieser in gewissem Sinne naturalistische Ansatz befähigt ihn, die Aporien der kontraktualistischen Staatstheorie, wie Hobbes

39 TP 7/1, am Beispiel der Monarchie. Oder kürzer: Es gilt, „daß jedes Rechtsgesetz der erklärte Wille des Königs ist, aber nicht jeder Wille des Königs auch Gesetz ist“ (TP 7/1). Das ist, in knappen Worten, die Lehre von der konstitutionellen Monarchie. Für die Demokratie fehlen wegen des fragmentarischen Charakters des TP die entsprechenden Ausführungen. 40 Die doppelte Bedeutung des Wortes „Empörung“, als Ausdruck der Mißbilligung aufgrund der imitatio affectuum in Form des Mitleids einerseits, des politischen, auf Ânderung der Herrschaftsverhältnisse gerichteten Aufstandes andererseits, ist also in Spinozas Philosophie erklärt. 
sie entwickelt hatte, dadurch zu vermeiden, daß er die implizit demokratische Konstitutionslogik staatlicher Souveränität, die auch bei Hobbes vorliegt, gegen deren „Aufhebung“ im Autorisierungsvertrag durchhält und daraus das Modell einer prozedural verflüssigten und konstitutionell begrenzten Herrschaft im Sinne der Demokratisierung des Staates entwickelt ${ }^{41}$.

Spinozas politische Philosophie stellt aber auch eine bestimmte Art der Anknüpfung an die kommunalistische Tradition dar, und zwar vor allem deshalb, weil er die kooperative Entstehung und Funktionsweise des Staates als das allen Regierungsformen zugrunde liegende, in der Demokratie am reinsten prozedural ausgeprägte und hier gegen absolute personale Herrschaft sperrige „Gesetz" der Politik entfaltet und in der rechtlichen Garantie von Bürgerfreiheit zugleich die besten Entfaltungsbedingungen für ein vernunftbestimmtes und daher wesentlich gemeinsames Leben sieht - ohne daß er sich doch der Einsicht in die Unaufhebbarkeit von Herrschaft unter der Bedingung zunächst und zumeist leidenschaftsbestimmten menschlichen Handelns und den daraus folgenden Erfordernissen einer starken Souveränität verschließen würde. Das zeigt sich auch darin, daß er an mehreren Stellen, u. a. in der Frage der konstitutionellen Begrenzung der personellen Herrschaft, auf von Althusius her vertraute Muster zurückgreift, ohne diese jedoch - und das ist der zentrale Punkt - mit nicht erzwingbaren inneren Konformitätsanforderungen zu belasten.

Damit sind Demokratisierung der Staatsorganisation, Konstitution einer gesamtstaatlichen zentralen Souveränität und rechtliche Ermöglichung eines weltanschaulichen Pluralismus auf hohem Abstraktionsniveau in einem einheitlichen prozedural akzentuierten Begründungsgang so miteinander verknüpft, daß diese Gestalt der politischen Philosophie, systematisch gesehen, das Niveau bezeichnet, hinter das eine sowohl moderne wie realitätsaffine Theorie nicht wird zurückfallen können.

\section{Jean-Jacques Roussean oder Die zweite Geburt des Menschen aus dem Vertrag}

6.1 Jean-Jacques Rousseau (1712-1762), der Herkunft nach Schweizer, dem hauptsächlichen Lebens- und Schaffensraum nach Franzose, hat nicht nur die Theoriegestalten von Althusius, Hobbes und Spinoza - sowie Pufendorfs und Lockes - aufgenommen, sondern auch die Erfahrungen der schweizerischen dörflichen ${ }^{42}$ und städtischen Bürgergemeinden nicht weniger als die eines hobbesia-

${ }^{41}$ Das gilt nicht nur für die Demokratie als Regierungsform, sondern wird, sehr detailreich und z. T. zeitbedingt, auch für Monarchie und Aristokratie ausgeführt. Die ersten Herausgeber haben, offensichtlich in Analogie zum von Spinoza selber festiggestellten TTP, dem TP als Untertitel beigegeben, daß in ihm "gezeigt wird, wie eine Gesellschaft mit monarchischer Regierung und wie eine mit aristokratischer Regierung einzurichten ist, damit sie nicht der Tyrannei verfällt und damit Friede und Freiheit der Bürger unangetastet bleiben "; und in der Tat geht die Intention Spinozas durchgehend dahin aufzuzeigen, daß Herrschaft, in welcher „Regierungsform“ auch immer, umso stabiler ist, je stärker in ihr Rückkoppelungsprozesse zwischen Regierenden und Bürgern institutionell verankert sind.

42 Vgl. seine überschwengliche Schilderung der lockeren Vergesellschaftung weitgehend aut- 
nisch geprägten Absolutismus in Frankreich „im Rücken“, als er sich, wiederum gut ein Dreivierteljahrhundert später, daran macht, die kontraktualistische Logik in ihrer freiheitsschaffenden und -verbürgenden Reinheit noch einmal, und zwar nunmehr gegen deren herrschaftsstabilisierenden Mißbrauch bei Hobbes und Pufendorf, aber auch bei Locke, voll zur Geltung zu bringen und so zu gestalten, daß er dabei zugleich dem spinozanischen Utopieverdikt gegenüber einer rein normativen, nicht in einer realistischen Anthropologie verankerten politischen Theorie entkommt.

6.2 In einer vor allem gegen Hobbes, aber auch gegen Locke gerichteten Konzeption des Naturzustandes beschreibt Rousseau diesen als einen Zustand der vollkommenen Herrschaftsfreiheit, die darin gründet, daß die Individuen ein asoziales, weil weitgehend quasi-instinktgeleitetes Leben in einer Umwelt führen, die genügend Ressourcen für die Befriedigung der elementaren, jeweils ad hoc auftretenden und so auch befriedigten Bedürfnisse bereitstellt. Daß der Mensch von Natur frei ist, ist der wichtigste Befund dieser Konzeption. Was dagegen die vorausgehenden Naturzustandstheoretiker als Naturausstattung der Menschen ausgegeben haben, ist die Sozialnatur des Menschen auf einer bestimmten, bereits antagonistisch gewordenen Stufe der Gesellschaftsgeschichte, so daß es nicht Wunder nimmt, wenn eben diese staatlich-herrschaftlich formierte Gesellschaft, der das Bild vom Naturzustand des Menschen entnommen ist, dann als Heilmittel angepriesen wird ${ }^{43}$.

Aufgrund nicht interner, entwicklungslogischer, sondern externer Umstände (Naturkatastrophen) sind die Menschen gezwungen, sich zu vergesellschaften. In so sich herausbildenden Formen einfacher naturwüchsiger Vergesellschaftung können sie solange relativ harmonisch koexistieren, wie ihr Streben nach Selbsterhaltung noch vom gleichfalls ursprünglichen Affekt des Mitleids (s. o. 5.4 zu Spinoza) in ihren gemeinschaftsschädlichen Ausdrucksformen in Zaum gehalten wird.

Erst als aufgrund der Arbeitsteilung sich Umfang wie Unterschiedlichkeit der verfügbaren Güter vergrößern und gleichzeitig auch die Bedürfnisse der Menschen vervielfachen, tritt eine Situation ein, in der sich - gleichzeitig mit einer starken ökonomischen und sozialen Differenzierung der Gesellschaft in Arme und Reiche - der "unschuldige“ amour propre elementarer Selbsterhaltungsbedürfnisse in einen egoistisch sich verschließenden, das Mitleid schwächenden und tendenziell abtötenden amour de soi-même verwandelt. Der erfolgreiche Versuch der Reichen, im Appell an das elementare Sicherheitsbedürfnis aller Gesellschaftsmit-

arker Bauernhöfe: Jean-Jacques Rousseau, Brief an Herrn d'Alembert..., in: ders., Schriften (München 1978) Bd. 1, $394 \mathrm{f}$.

${ }^{43}$ "Sie (d.i. alle vorangegangenen Naturrechtstheoretiker. Wa) sprachen vom wilden Menschen und beschrieben den bürgerlichen Menschen": Jean-Jacques Rousseau, Diskurs über die Unglcichheit / Discours sur l'inégalité, hrsg. von Heinrich Meyer (Paderborn 1984, zuerst 1755) $69,71$. 
glieder zu einer von allen akzeptierten Eigentumsrechtsordnung zu gelangen ${ }^{44}$, binden auch die Armen an diese Ordnung ungleicher Eigentumsverhältnisse unter dem Deckmantel der formellen Rechtsgleichheit und Rechtssicherheit und reproduziert und potenziert damit die vorhandenen Abhängigkeitsverhältnisse. Es tritt eine Lage ein, in der wechselseitige Abhängigkeit voneinander für die Befriedigung der meist künstlich hervorgerufenen Bedürfnisse und eine egoistisch verformte Affektstruktur sich wechselseitig blockieren ${ }^{45}$.

6.3 Damit ist gesellschaftssystematisch ein höchst instabiler Zustand erreicht, in dem, wie Rousseau in doppelter Negation formuliert, „die Hindernisse, die ihrem (d.i. der Menschen. Wa.) Fortbestehen im Naturzustand (!) schaden, in ihrem Widerstand den Sieg davontragen über die Kräfte, die jedes Individuum (!) einsetzen kann, um sich in diesem Zustand zu erhalten “46.

Erst jetzt, wo sich Sprache, Vernunft und ein differenziertes, aber zugleich die $\mathrm{Na}$ tur des Menschen entstellendes Affektleben entwickelt haben, ist es möglich, aber zugleich erforderlich geworden, daß die Menschen aus dem Prozeß naturwüchsiger, immer mehr herrschaftsüberformter Vergesellschaftung ausbrechen und sich zu einer neuen Form gesellschaftlicher Einheit bewußt und frei zusammenschließen. Erst jetzt hat die Stunde des Gesellschaftsvertrages geschlagen, alles andere ist bloße Vorgeschichte bewußter Vergesellschaftung.

Rousseau stellt an diejenige Form des gesellschaftlichen Bandes, welches die Menschen sich selber freiwillig anlegen, höchste Anforderungen:

Die zu bildende Form der gesellschaftlichen Einheit muß so beschaffen sein, daß (1) darin kein Mensch über andere herrscht, insofern also eine Wiederherstellung der ursprünglichen Herrschaftsfreiheit des Naturzustandes statthat, - auch wenn die Autonomie des Individuums im Sinne der vollständigen Unabhängigkeit von anderen nicht restituierbar ist ${ }^{47}$;

(2) die Interessen der Menschen, die sie im nach Rousseau vorgesellschaftlichen Zustand, also in der Konkurrenzgesellschaft, entwickelt haben, sofern sie mit der

\footnotetext{
44 Auf diesen Aspekt hat Kersting, (wie Anm. 2), 142-147, nachdrücklich hingewiesen. Hier ist auch der Ort, an dem nach Rousseaus Meinung Locke steht.

45 "(N)os besoin nous rapprochent à mésure que nos passions nous divisent", heißt es knapp und präzise im Genfer Manuskript des Contrat social:Jean-Jacques Rousseau, Du contrat social (Manuscript de Genève), in: ders., Oeuvres complètes, ed. de la Pleiade, vol. 3, (Paris 1964) 282, Zeilen 8-9.

46 Jean-Jacques Rousseau, Gesellschaftsvertrag oder Grundsätze des Staatsrechts (Stuttgart 1977) im folgenden zit.: CS, nach Buch/Kapitel: Seite 1/6: 16.

${ }^{47}$ Deshalb beschreibt Rousseau auch gleich zu Beginn des CS (1: 5) das Problem so, daß er nachweisen will, was den „Ketten“, in denen der Mensch nach dem Ausgang aus dem Naturzustand liegt, „Rechtmäßigkeit“ verleihen könne. Insofern ist das im Staat des Contrat Social herrschende Recht Vernunftrecht und nicht ursprüngliches Naturrecht. Darin nimmt Rousseau die Einsicht auf, die sowohl Hobbes als auch, in einer Rousseau näheren Art und Weise, Spinoza formuliert hatte (z. B. TP 3/3), daß alles wirkliche Recht auf Kooperation beruhendes, also gesellschaftlich erzeugtes Recht ist. Vgl. auch den Hinweis bei Kersting, (wie Anm. 2), $150 \mathrm{f}$.
} 
neuen Einheit vereinbar sind, gewahrt und gesichert sind;(3) daß es sich um eine stabile, d. h. von allen affirmierte und getragene Einheit handelt.

Rousseau beschreibt daher die Anforderungen an die gesuchte Problemlösung zusammenfassend so: „Finde eine Form des Zusammenlebens, die mit ihrer gesamten gemeinsamen Kraft (Moment 3. Wa.) die Person und das Vermögen jedes einzelnen Mitgliedes verteidigt und schützt (Moment 2. Wa.) und durch die doch jeder, indem er sich mit allen vereinigt, nur sich selbst gehorcht und so frei bleibt wie zuvor (Moment 1! Wa.)" (CS 1/6: 17). Rousseaus Lösung ist ein spezifisch gefaßter Vertrag, den jeder mit allen anderen schließt: „Gemeinsam stellen wir alle, jeder von uns, seine Person und seine ganze Kraft, unter die oberste Richtschnur des Gemeinwillens [volonté générale]; und wir nehmen, als Körper, jedes Glied als untrennbaren Teil des Ganzen auf" (CS 1/6: 18).

Darin sind die angeführten Bedingungen wie folgt erfüllt:

(1) Indem alle gemeinsam über die Formen des Zusammenlebens entscheiden, muß sich niemand dem Diktat eines anderen beugen, ist also personale Herrschaft ausgeschlossen; es handelt sich um eine vollständige Reziprozität von Willensverhältnissen, in die keine der vorher vorhandenen Machtpositionen eingeht. Insofern „bleiben“ alle frei - so frei nämlich, wie sie als autonom den Kooperationsvertrag schließende Individuen waren.

(2) Da alle freiwillig nur das beschließen, was sie als ihren Interessen dienlich ansehen, und jeder seine Interessen selber am besten kennt, kann nur solches beschlossen werden, was auch diesen Interessen gerecht wird. Und zugleich gewinnen alle, als einzelne, die vereinigte Willens- und Handlungsmacht des gesamten gesellschaftlichen Körpers als den Garanten, der „die Person und das Vermögen“ - das ihnen gemäß dem gemeinsamen Beschluß zukommt - „verteidigt und schützt".

(3) $\mathrm{Da}$ es sich nicht mehr um einander ausschließende, in Konkurrenz zueinander stehende Interessen handelt, ist die neue Einheit zugleich stabil, da es einen breiten Bereich der substantiellen Übereinstimmung der Einzelwillen gibt, der sich selbst reproduziert.

Das Resultat der gemeinsamen Willensbildung sind die allgemeinen Regeln des Zusammenlebens, nach denen jedem das Seine bestimmt wird, also allgemeine Gesetze. Und das durch diesen Zusammenschluß sich konstituierende Volk ist seinerseits souverän und an nichts gebunden als an das, was es selber beschließt - und solange es diesen Beschluß nicht revidiert. Rousseau geht dabei so weit, daß er auch diese Form der egalitär-demokratischen politischen Willensbildung als durch Beschluß jeweils zu erneuernde versteht.

6.4 Daraus ergibt sich dann die institutionelle Struktur des Staates ganz einfach:

- Die Gesetzgebung ist immer und nur Sache der zum corps politique zusammengeschlossenen Kontrahierenden, d. h. des souveränen Volkes, das daher auch nicht repräsentierbar ist; und da der Gemeinwille definiert ist als derjenige, der das von allen gemeinsam Gewollte in die Form allgemeiner Gesetze gießt, kann er auch nicht irren. 
- Die Exekution der Gesetze und folglich die Kontrolle der Bürger bezüglich der Einhaltung der Gesetze ist das Geschäft der Regierung, die daher immer nur als gesetzesgebundene legitim arbeitet. Sobald die Regierung dagegen die Gesetzesbefugnis usurpiert, wird die Regierung despotisch; und tyrannisch ist eine zwar gesetzeskonforme, aber nicht vom Souverän eingesetzte Instanz (CS 3/ 10). In beiden Fällen hat der Souverän, haben auch einzelne Angehörige des souveränen gesetzgebenden Körpers, quasi subsidiär, das Recht und die Pflicht zum Widerstand.

6.5 Rousseaus politische Philosophie, die er im „Contrat Social“ entwickelt, ist einer der großen Versuche, die Realisierbarkeit des Modells einer in Autonomie von den Menschen erzeugten, herrschaftsfreien, ethisch bestimmten Lebensführung, wie sie der kommunalistischen Erfahrung vorschwebt, im Maßstab des modernen Territorialstaates mit ausdifferenzierten Lebenssphären nachzuweisen. Dabei bildet die von Spinoza explizit gemachte Einsicht, daß die Macht des Staates die Macht der Menge ist, ebenso wie die daraus von Spinoza gezogene demokratische Konsequenz ein besonders wirkungsmächtiges Denkmotiv ${ }^{48}$. Rousseau entfaltet die demokratischen Implikationen des kontraktualistischen Gedankens in Reinkultur. Gleichzeitig insistiert er aber, wie das schon die Betonung des Desiderats (2) erkennen läßt, wiederum in der Sache mit Spinoza, auf dem handlungs-, und d. h. dem affekttheoretischen Ausweis der von ihm konzipierten egalitär-freiheitlichen Gesellschaft - und scheitert an eben diesem Ausweis ${ }^{49}$ :

a) Schon im Vorspann des 1. Buches setzt Rousseau sich zum Ziel, eine Lösung des Problems antagonistisch gewordener gesellschaftlicher Verhältnisse auszuarbeiten dergestalt, „daß das, was Recht zuläßt, stets mit dem verbunden (sei), was der Vor-

${ }^{48}$ Während die Art und Weise, in der die politischen Theorien von Hobbes, Pufendorf, Locke bei Rousseau präsent sind, vielfach Aufmerksamkeit gefunden hat - vgl. dazu z. B. Robert Derathé, Jean-Jacques Rousseau et la science politique de son temps (Paris 1988), und Reinhard Brandt, Rousseaus Philosophie der Gesellschaft (problemata 16, Stuttgart-Bad Cannstatt 1988), ferner die zahlreichen Arbeiten zum Verhältnis Rousseaus zu jeweils einem einzelnen der genannten Denker -, ist das nach der hier vorgelegten Interpretation besonders relevante Verhältnis zu Spinoza bisher monographisch nicht behandelt worden; vgl. einstweilen Madeleine Francès, Les reminiscences spinozistes dans le contrat social de Rousseau, in: Revue philosophique de la France et de l'Etranger (1951) 60-84, die in der systematischen Bilanz der spinozanischen Theorie den Vorzug gibt. Die Gegenüberstellung einzelner signifikanter Passagen aus Spinoza und Rousseau ist als Anhang beigefügt.

49 Wolfgang Kersting kritisiert das Insistieren Rousseaus auf dem interessen-, also affekttheoretischen Ausweis der Möglichkeit solch substantieller Einheit des Gesellschaftskörpers als inkonsistente Verunreinigung des kontraktualistischen Arguments (Kersting [wie Anm. 2], $167 \mathrm{ff}$.). Wenn er am Schluß seiner Monographie dann aber - in der aufgrund der kommunitaristischen Kritik an Rawls von diesem selber revidierten Fassung des kontraktualistischen Arguments (352-355) - den Kontraktualiusmus als in einer bestimmten, eben der westlichen, Tradition und Lebensform gegründete Rechtfertigungstheorie politischer Herrschaft versteht, so rekurriert er selber der Sache nach auf einen Faktor, der, wie er einräumt, den kontraktualistischen Gedanken transzendiert. Das aber heißt, daß Rousseaus Beweisprogramm - von dem ich unterstelle, daß es, auch, von Spinoza beeinflußt ist -, weiter aktuell ist, auch wenn Rousseaus eigener Lösungsversuch daran gescheitert ist. 
teil vorschreibt, damit Gerechtigkeit und Nutzen nicht getrennt gefunden werden " (CS: 5) ${ }^{50}$. Das Interesse der einzelnen Kontrahierenden ist durch die gesetzgebende Kompetenz des gemeinsamen Körpers aber nur dann nicht verletzt, wenn dieser nur beschließt, was den Interessen aller entspricht. Das setzt nun aber, was den Umfang der legitimen Gesetzgebung betrifft, voraus, daß die Interessen der Kontrahierenden weitgehend übereinstimmen, also identisch oder miteinander kompatibel sind ${ }^{51}$. Sind die Lebensverhältnisse und damit die Interessen der Bürger nicht weitgehend homogen, nehmen die Sonderinteressen, die nach Rousseau ihren legitimen Ort haben, zu, so verdünnt sich im selben Maße das Konkretgemeinsame der Willen, der Staat wird geschwächt.

Deshalb postuliert Rousseau konsequent: „Dieser Übergang vom Naturzustand zum bürgerlichen Zustand erzeugt im Menschen eine sehr bemerkenswerte Veränderung, weil dadurch in seinem Verhalten die Gerechtigkeit“ - in Form der Zustimmungsbereitschaft zum allgemeinen, d. h. gemeinwohldienlichen Gesetz „an die Stelle des Instinktes" - nämlich des blinden Triebes der Selbsterhaltung "tritt und seinen Handlungen diejenige Moralität" - als gewußte und gewollte Pflichtbindung seines Handelns anstelle des instinktartig wirkenden Mitleids "verliehen wird, die ihnen zuvor mangelte" (CS 1/8: 22).

Die Einsicht, daß nur das Überwiegen der Gemeinwohlorientierung den gesellschaftlichen Körper kraftvoll und handlungsfähig macht, ist nur die andere Seite des Problems der Homogenität der Lebensverhältnisse: Beide reproduzieren sich wechselseitig. Das Wissen darum, daß solches Überwiegen der Gemeinwohlorientierung einer affektiven Stützung bedarf, um jederzeit handlungswirksam zu sein, veranlaßt Rousseau so, ganz konsequent, dazu, eine „religion civile“ zu konzipieren, die als Patriotismus eben diese Orientierung am Gemeinsamen auch gefühlsmäßig stützt, und Rousseau geht so weit, dabei für diejenigen Bürger die in ihrem Handeln gegen diese Bürgerreligion verstoßen, die Todesstrafe vorzusehen $(\mathrm{CS} 4 / 8)^{52}$.

Das Problem der affektiven Stützung der Gemeinwohlorientierung, das durch die religion civile für die nicht Einsichts- und Vernunftfähigen gelöst werden soll, stellt sich für Rousseau deshalb in besonderer Schärfe - und es spricht für die Aufrichtigkeit seines Denkens, daß er selber eindringlich darauf hinweist -, weil er die neue Gesellschaft des „Contrat Social“ als nur radikalen Bruch mit der Vorge-

50 Die Zweigleisigkeit der Beweisführung, einer interessenökonomischen und einer universalistisch-kontraktualistischen, und das Scheitern der Vermittlung beider, hat Maximilian Forschner, Rousseau (Alber Kolleg Philosophie, Freiburg, München 1977) 120-157, herausgearbeitet. Vgl. auch den entsprechenden Abschnitt bei Kersting, (wie Anm. 2), $167 \mathrm{f}$.

51 Wenn Rousseau, außer beim Abschluß des Vertrages selber, nicht Einstimmigkeit fordert, sondern die Geltung des Mehrheitsvotums vertritt, so begründet er das damit, daß sich so, indem die Zahl der Nichtzustimmenden und eine entsprechende Zahl der Zustimmenden sich gegenseitig neutralisieren, eben der Überschuß der Mehrheit als das tragfähige Gemeinsame übrigbleibt.

52 Vgl. dazu Karl Dietrich Erdmann, Das Verhältnis von Staat und Religion nach der Sozialphilosophie Rousseaus (Der Begriff der "religion civile“) (Historische Studien 271, Berlin 1935) und Walther, (wie Anm. 57). 
schichte möglich konzipiert. Damit ist aber die Möglichkeit abgeschnitten, daß der Staat auf die „im Schoße der alten Gesellschaft“ sich herausgebildeten Motivationen und Dispositionen zurückgreifen kann ${ }^{53}$. Diese Kombination kontraktualistischer mit handlungs- oder affekttheoretischen Erwägungen, in der, analytisch gesehen, die Stärke des Rousseauschen Beweisprogramms liegt, dieser „äquivoke Kontraktualismus, der Rechtsfiguren und Ethosformen ineinander schiebt", erweist sich als notwendige Bedingung für die Realisierung des egalitär-demokratischen Projekts. Er „wird zum Sinnbild einer ethischen Metamorphose, einer Verwandlung der natürlichen Menschen in Gemeinschaftswesen, einer Transformation natürlicher Lebensverhältnisse in eine moralische Welt ${ }^{454}$.

b) Rousseau ist sich der Gefährdetheit dieses seines Projekts durchaus bewußt. So spricht er davon, daß jede politische Körperschaft, so gut wie jeder Mensch, den Gesetzen des Entstehens und des Vergehens unterworfen ist, daß sie „die Keime ihrer Zerstörung in sich (trägt)“. Das ist deshalb so, weil „die Verfassung des Menschen ein Werk der Natur, die des Staates aber ein Werk der Kunst" ist. Zwar gilt: „Je besser der Staat verfaßt ist“ - je stärker also Interesse und Pflicht koinzidieren und damit die ethisch bestimmte Lebensweise institutionell gestärkt wird und sich die Präferenzstruktur des Individuums transformiert -, „desto mehr überwiegen im Herzen der Bürger die öffentlichen Angelegenheiten die privaten " (CS 3/15: 102). Aber da die allgemeinen Gesetze der Menschennatur - man denkt hier unwillkürlich an Spinozas Lehre von der Naturgesetzlichkeit des Affektlebens durch die Staatsbildung nicht aufgehoben werden, ist es unvermeidlich, daß im Laufe des Staatslebens der Patriotismus erkaltet, die Privatinteressen sich in der Handlungspräferenz der Bürger immer stärker geltend machen, wozu auch die „Übergröße der Staaten“ beiträgt (CS 3/15), so daß schließlich der Vorrat an substantiell gemeinsamer Orientierung sich abschwächt und damit auch die Gesetze ihre Kraft zu verlieren beginnen, bis schließlich der politische Körper an Lebenskraft verliert und schließlich zerfällt.

Was Rousseau hier noch als staatsbiologisch bedingten Verfall analysiert, daß nämlich die Realisierungsbedingungen seiner Lösung im Zuge der jeweiligen Gesellschaftsgeschichte dahinzuschwinden beginnen, das hat er später, anders als die meisten Rousseauisten, auch direkt und systematisch ausgesprochen: daß er nämlich mit seiner Lösung gescheitert ist.

In einem Brief an Mirabeau vom 26.7. 1767, also fünf Jahre nach der Veröffentlichung des "Contrat Social", schreibt er, im Anschluß an das dort gestellte Beweisziel, eine neue Form der gesellschaftlichen Einheit zu finden: „Wenn diese

53 Da denkt Marx später realistischer, wenn er darlegt, daß sich die neue Gesellschaft nur etablieren kann, wenn sie als Selbstbewegung der alten Gesellschaft über sich hinaus entsteht, sich also nur aus der jeweils fortgeschrittensten Gestalt der alten Gesellschaft entwickeln kann, in der sich bereits, wenn auch noch durch die alte Gesellschaftsstruktur "gefesselt", die Produktivkräfte der neuen Gesellschaft soweit entfaltet haben, daß eine gewisse Anschließbarkeit vorhanden ist - was dann realgeschichtlich nicht eingelöst wurde, mit dem bekannten Ergebnis.

54 Kersting, (wie Anm. 2), 168. 
Form unglücklicherweise nicht auffindbar ist, und ich glaube, daß sie nicht auffindbar ist, bin ich der Ansicht, daß man zum anderen Extrem übergehen muß und mit einem Schlag die Menschen so sehr unter das Gesetz stellen muß, wie das nur möglich ist; folglich den willkürlichen Despotismus errichten, und zwar den willkürlichsten, der möglich ist; ich wollte, der Despot könnte Gott sein. ${ }^{\text {" } 55}$ Jene „vollkommene Entäußerung“ der Menschen an den politischen Körper, die zur Lebensfähigkeit desselben erforderlich ist, existiert nun auch nach Rousseaus Urteil "mehr in der Einbildung als in der Wirklichkeit“ (Spinoza, TP 2/15).

So begreift Rousseau denn am Ende die politische Lösung des Problems selber eher als Abgesang auf eine unwiederbringlich vergangene Epoche der Menschheitsgeschichte und erprobt für sich andere Lösungen dafür, wie ein „unbeschädigtes Leben im beschädigten" (Adorno) möglich sein könnte: in ethisch-religiöser Fundierung eines autbentischen Lebens inmitten einer verfallenen Umwelt im Erzieher-Zöglingsverhältnis und weitgehender dadurch ermöglichter Innenstabilisierung; in einem Leben rücksichtsloser Aufrichtigkeit vor sich selbst und vor der Welt; in einem Leben der Einsamkeit fern aller Sozialität ${ }^{56}$ - alles ,individualistische“, „unpolitische" Lösungen.

\section{Rückblick und Ausblick}

7.1 Blicken wir auf die betrachteten Gestalten einer Rechts- und Staatstheorie zurück, von denen zumindest die drei zuletzt Behandelten gemeinsam haben, daß sie Rechtsordnung und Staatsgewalt allein aus der Interaktion der Menschen selber herzuleiten versuchen - und daher einer Begründung von Recht und staatlicher Herrschaft "von oben", als Setzung einer menschen- und weltüberlegenen jenseitigen Macht, entgegenarbeiten -, so ergibt sich am Leitfaden des Problems, wie die Errungenschaft kommunalistischer politischer Selbstorganisation im Zuge der Begründung einer komplexen ausdifferenzierten modernen Gesellschaft bewahrt werden kann, Folgendes:

a) Althusius gehört nicht in den Zusammenhang der modernen Gesellschaftstheorien, da sein Modell des Politeuma, der politischen consociatio symbiotike, kein unmittelbares Konstitutionsverhältnis zwischen den einzelnen Gesellschaftsmitgliedern und der gesamtstaatlichen Herrschaft kennt. Stattdessen rezipiert er das aristotelische Modell einer stufenweisen Assoziation von jeweils als vorausgehend angesehenen sozialen Formen und modifiziert es nur insofern, als er zwischen dem aristotelischen Dorf und der Polis noch die Provinz einfügt. Das Festhalten

55 Jean-Jacques Rousseau, The Political Writings, vol. 2, ed. by E.C. Vaughan (Oxford 1969) 160 f., zit. nach Stefan Breuer, Sozialgeschichte des Naturrechts (Beiträge zur sozialwissenschaftlichen Forschung 42, Opladen 1983) 445; Übers. u. Hervorh. von mir.

56 Vgl. die Charakterisierung der vier "Auswege“ aus der Aporie des politischen Lebens der Gegenwart, wie sie Günter Figal, Die Rekonstruktion der menschlichen Natur: Zum Begriff des Naturzustandes in Rousseaus "zweitem Discours", in: Rousseau und die Folgen (Neue Hefte für Philosophie 29, Göttingen 1989) 24-38, skizziert hat. 
an der Einheit von Glaubens- und Bürgergemeinde der reformierten theologischen Tradition ermöglicht es ihm zwar, das Volk als Träger auch der gesamtstaatlichen Souveränität festzuhalten und die Herrschaftsgewalt als Auftragsverhältnis unter festgelegten rechtlichen Bedingungen (leges fundamentales), also als Rechtsverhältnis, zu bestimmen; das ist die Form, in der sich das kommunalistische Moment bis in die oberste gesellschaftlich-politische Ebene hinein durchhält. Indem er aber zugleich jede politische Einheit mit dem Erfordernis einer sittlich und religiös einheitlichen Lebensführung befrachtet, wie es nur in kleineren sozialen Gruppen, wenn überhaupt, durchhaltbar ist, überlastet er seine politische Theorie in einer Weise, welche dem schon einsetzenden Prozeß der religiösen und ökonomischen, auch sozialen, Differenzierung nur um den Preis einer Regression hätte standhalten können - und es blieb dem 20. Jahrhundert vorbehalten, solche Regressionsgestalten in Form politischer Theorien auf deutschem Boden hervorzubringen.

b) Hobbes entwickelt das Grundmuster der modernen Gesellschaftsvertragstheorie, derzufolge politische Herrschaft nur dann legitim ist, wenn sie als Resultat der freiwilligen Selbstbindung der Bürger, als Resultat eines zwischen allen Gesellschaftsmitgliedern geschlossenen Vertrages, explizierbar ist. In der von ihm angebotenen Lösung verschwindet jedoch die vorausgesetzte Autonomie der kontraktierenden Individuen fast spurlos in dem Resultat, dem ein für allemal authorisierten absoluten Souverän; fast spurlos ist dieses Verschwinden, weil in dem nicht aufgebbaren Recht der Selbstverteidigung gegen den lebens- und freiheitsbedrohenden Zugriff des Souveräns ein Kern vorstaatlicher Menschenrechte festgehalten ist, der aber zugleich geeignet ist, das ganze Gebäude zu erschüttern.

c) Spinoza fällt aus dem Rahmen neuzeitlicher Vertragstheoretiker, zu denen der Oberflächengestalt nach auch Althusius gehört, ganz heraus. Seine Strategie, sowohl die Einsicht in vorstaatliche Sozialbeziehungen als auch die kommunalistische Erfahrung der Stärke durch Kooperation zu bewahren, ohne die Überlastung mit ethischen und religiösen Pflichten sich aufzuhalsen, geht schon vom Ansatz her einen anderen Weg: Er setzt nämlich - und zwar fast gegen die gesamte Tradition -, politische und soziale Freiheit im Sinne der Handlungsfreiheit nicht als vorsoziale und vorstaatliche Gegebenheit voraus, sondern zeigt, daß Handlungsfreiheit zu allererst das Resultat von Kooperationsbeziehungen ist, die in rechtlichen Institutionen ihren Niederschlag finden und nur als herrschaftsgestützte auch auf Dauer Wirksamkeit gewinnen, wobei die Herrschaft jedoch immer von der Akzeptanz der Beherrschten als der causa immanens jeder Regierungsgewalt genetisch und damit auch faktisch abhängig ist. Genau aus dieser Einsicht in die Struktur aller politischen Herrschaft als eines nicht stillstellbaren Machtkreislaufes, also aus einer empirischen Tatsache und nicht aus einem normativen Postulat, demonstriert er dann die Überlegenheit der Demokratie über andere Regierungs-, also Herrschaftsformen und die herrschaftsstabilisierende, aber zugleich auch herrschaftsbegrenzende Funktion von individuellen Freiheitsrechten mit der Meinungsäußerungsfreiheit und der Wissenschaftsfreiheit an der Spitze. Hier ist also 
die kommunalistische Erfahrung zum Grundmuster der „Logik des Politischen“ geworden und zugleich als herrschaftslimitierende, freiheitskonstitutive Theorieoption wirksam. Vollständige Herrschaftsfreiheit kann in einer solchen Konzeption nur als Versuch erscheinen, die Naturzugehörigkeit und Natureingebundenheit des Menschen voluntaristisch zu überspringen - mit jener Dialektik, die wir kennen. Erst im Rahmen einer solchen rechtlich gestalteten Freiheit individueller Lebensführung kann sich dann auch ein ethisch selbstbestimmtes Leben, auch in freiwilliger Gemeinschaft, dauerhaft konstituieren.

d) Rousseau schließlich unternimmt den gewaltigen Versuch, auf der Basis und mit den Mitteln der modernen Gesellschaftsvertragstheorie zugleich eine ethisch und wo die Einsicht nicht ausreicht, auch religiös - bestimmte Lebensgemeinschaft auf gesamtgesellschaftlicher Ebene in Überwindung der interessenzerklüfteten modernen Zivilisationsgesellschaft zurückzugewinnen. Hier liegt also der Versuch vor, jenes kommunalistische Modell einer von allen geteilten sittlich bestimmten Lebenswelt auf dem Boden der Moderne, d. h. auf dem Boden der unaufgebbaren Autonomie des Individuums, zurückzugewinnen. In dem Maße jedoch, wie die Vertragspartner als empirische Subjekte noch von den Sozialisationsschäden der Konkurrenzgesellschaft gezeichnet sind, muß der Staat auf eine auf Unterwerfung statt auf freie Anerkennung ausgehende Staatsreligion, die damit das Freiheitsniveau des Christentums notwendig unterschreiten muß, zurückgreifen; kurz: In der Durchsetzungsphase - von dem Problem ihrer Etablierung durch den législateur als einen Deus ex machina ganz abgesehen - muß der Staat des contrat social zu einer Herrschaftsform regredieren, die Rousseau zur Einsicht in die Unrealisierbarkeit seines Projektes und zum Durchspielen nunmehr individualistischer, unpolitischer Lösungen des Problems eines „unbeschädigten Lebens" veranlaßt.

7.2 Die Behauptung, daß das - noch dazu aufgeklärte - Eigeninteresse der Individuen nicht auf Dauer jene Motivation zu erzeugen vermag, welche politische Institutionen des freien und gemeinsamen Lebens aufrecht erhält, bildet den Ausgangspunkt des Kommunitarismus. Daher versucht er in seiner Liberalismuskritik, dessen Einseitigkeit und dessen Mangel an Tragfähigkeit für eine lebensfähige politische Synthesis der Gesellschaft zu überwinden und Vorschläge für die Ausgestaltung eines ergänzenden Spektrums affektiv wirksamer, ethisch bestimmter Verstärkungen der politischen Einheit zu entwickeln. Schon im Namen ist die Verwandtschaft mit dem Kommunalismus erkennbar. Dabei ist sich diese liberalismuskritische Bewegung vor allem in den USA sehr wohl der Gefahr bewußt, welche in einer ethisch und/oder religiös aufgeladenen Kritik der modernen, repräsentativdemokratischen Gesellschaft liegen kann - angesichts des in der US-Gesellschaft weit verbreiteten religiösen Fundamentalismus ebenso relevant wie angesichts der Karriere auf Tönnies sich berufenden Gemeinschaftsgedankens in Deutschland.

So soll ein kurzer Hinweis auf Richtung und Gestalt des Kommunitarismus diese Überlegungen abschließen und zugleich die Aktualität der kommunalistischen Erfahrung in der gegenwärtigen politischen Theorie belegen: Das Thema ist 
noch nicht erledigt, weil jene Erfahrungen als immer weniger im politischen Leben der heutigen Demokratien „aufgehoben“, ja weil sie geradezu als hochgefährdet erscheinen.

Die zuerst von Michael J. Sande ${ }^{57}$ formulierte Kritik am politischen Liberalismus besagt, daß

- das ungehemmte Fortschreiten der Individualisierungstendenz der - amerikanischen - Gesellschaft ein Klima der sozialen Kälte und der Rücksichtslosigkeit erzeuge und damit das ,gesellschaftliche Band" zu sprengen drohe,

- und daß der politische Liberalismus als die herrschende politische Philosophie dafür verantwortlich zu machen sei.

Was dabei auf der Strecke bleibe, sei das Bewußtsein, daß die modernen Freiheitsrechte, die Sandel ebenso erhalten will wie der von Sandel in erster Linie attackierte John Rawls ${ }^{58}$, nur dann diese Freiheit auch tatsächlich zu geben vermöchten, wenn es gelinge, gemeinschaftsbildende Sozialmilieus zu stabilisieren und zu kräftigen, da nur im Rahmen vorgängiger Sozialität und entsprechender Einstellungen der einzelnen der Umschlag freiheitssichernder Individualrechte in Solipsismus und soziale Kälte und Rücksichtslosigkeit vermieden werden könne. Was die Kommunitaristen - später auch u. a. Charles Taylor, von Hegel herkommend, und MacIntyre, der aristotelisch geprägt ist - einzuklagen suchen, ist also die Stärkung jener Erfahrungen fraglos geteilter Lebenswelten, die den metaindividualistischen Rahmen und zugleich die affektiven Ressourcen bereitstellen, um von den Freiheitsrechten einen anderen als selbstzerstörerischen Gebrauch zu machen. Hier kann man also sowohl das Althusianische Motiv der consociatio politike (als dessen Form des Aristotelismus) als auch Rousseaus „zweite“, ethisch bestimmte und auf Identifikation mit dem Gemeinwesen ausgerichtete Natur der den Souverän bildenden Staatsbürger wiedererkennen.

Diese Skizze mag genügen, um deutlich zu machen, inwiefern die Erfahrungen gemeinsamen Lebens als eine oder die wesentliche Bedingung dafür angesehen wird, daß der demokratische Egalitarismus sich nicht selber zerstört. In Deutschland ist diese Reflexion im Rahmen einer Analyse der angeblich politisch dysfunktionalen Folgen des Säkularisierungsprozesses von Wolfgang Böckenförde formuliert und zugleich als Apologie der Religion ausgemünzt worden, wenn er schreibt: „Der freiheitliche, säkularisierte Staat lebt von Voraussetzungen, die er selber nicht garantieren kann. ${ }^{* 59}$

57 Liberalism and the Limits of Justice (Cambridge u. a. 1982 u.ö.).

${ }^{58}$ Eine Theorie der Gerechtigkeit (Frankfurt a.M. 1975 u.ö.; zuerst amerikan. 1971). Diese Kritik hat Rawls zu einer Modifikation seiner politischen Theorie veranlaßt, an die wiederum Kersting, (wie Anm. 2), bes. 352-355 anschließt.

59 Ernst Wolfgang Böckenförde, Die Entstehung des Staates als Vorgang der Säkularisation, in: ders., Staat, Gesellschaft, Freiheit: Studien zur Staatstheorie und zum Verfassungsrecht (Frankfurt a.M. 1976; der Aufsatz zuerst 1967) 42-64 (60). Mit dieser These von der bleibenden Angewiesenheit der politischen Synthesis auf Religion habe ich mich beschäftigt in: Die Religion des Bürgers - eine Aporie der politischen Kultur der Neuzeit? Hobbes, Spinoza und Rousseau oder Über die Folgelast des Endes der politischen Theologie, in: Bürgerschaft und Bürgertugend (wie Anm. 10) (im Druck). 
Wie diese Stoßrichtung der Debatte sich gegenüber der Gefahr immunisieren kann, in die in Deutschland traditionell eingefahrenen und erst seit kurzem - mit Blick des Historikers betrachtet - verlassenen Bahnen eines antiindividualistischen Gemeinschafts- und Pflichtdenkens à la Pufendorf und Wolff, und später der Gemeinschaftsideologien des 20. Jahrhunderts, hineinzugleiten - in den USA wäre das die Moral Majority-Bewegung etwa eines Jerry Falwell -, das ist das Problem, zu dem die oben dargestellten Modelle politischer Einheitsbildung auf ihre Ergiebigkeit für die gegenwärtige Debatte hin erneut zu befragen wären. 


\section{Exkurs zu Entsprechungen in der Gesellschaftstheorie Spinozas und Rousseaus Textvergleich}

\section{Spinoza}

Wenn zwei zugleich zusammenkommen und ihre Kräfte verbinden, vermögen sie zugleich mehr und haben folglich zugleich mehr Recht auf die Natur als jeder von ihnen allein, und je mehr ihre Bedürfnisse so miteinander verbinden, desto mehr Recht werden alle zugleich haben. (TP $2 / 13$ )

$\mathrm{Da}$ aber im Naturzustand... jeder so lange unter eigenem Recht ist, solange er sich vor Unterdrückung durch einen anderen bewahren kann, und da einer allein sich vergebens vor allen zu wahren strebte, so ist das natürliche Recht des Einzelnen, solange es nur das des Einzelnen ist und durch seine Macht bestimmt wird, gleich Null...

Wir schließen daraus, daß von Naturrecht als dem Menschengeschlecht eigen doch eigentlich nur da die Rede sein kann, wo die Menschen gemeinsame Rechte haben.

(TP 15/2)

Auf diese Weise also kann sich ohne irgendwelchen Widerspruch gegen das natürliche Recht eine Gesellschaft bilden, und jeder Vertrag kann in vollkommener 'Treue gehalten werden; es braucht eben nur jeder die ganze Macht, die er besitzt, auf die Gesellschaft zu übertragen... Das Recht einer solchen Gesellschaft heißt Demokratie; sie ist demnach zu definieren als eine allgemeine Vereinigung von Menschen, die in ihrer Gesamtheit das höchste Recht zu allem hat, was sie vermag.

(TTP 16: 193, 19-22 u. 24-26/PhB 237, 3137 u. 39-238,2)

Damit glaube ich die Grundlagen einer demokratischen Regierung hinlänglich klar dargelegt zu haben. Ich habe diese lieber als alle anderen behandelt, weil sie, wie mir scheint, die natürlichste ist und der Freiheit, welche die Natur jedem einzelnen gewährt, am nächsten kommt. Denn bei ihr überträgt niemand sein Recht derart auf einen anderen, daß er selbst fortan nicht mehr zu Rate gezogen wird; vielmehr überträgt er es auf die Mehrheit der gesamten Gesellschaft, von der er selbst ein Teil ist. Auf diese Weise bleiben alle gleich, wie sie es vorher im $\mathrm{Na}$ turzustand waren.

(TTP 16: 195, 14-21/PhB 240, 12-21)

\section{Rousseau}

$\mathrm{Da}$ die Menschen nun keine neuen Kräfte hervorbringen, sondern nur die vorhandenen vereinen und lenken können, haben sie kein anderes Mittel, sich zu erhalten, als durch Zusammenschluß eine Summe von Kräften zu bilden, stärker als jener Widerstand, und diese aus einem einzigen Antrieb einzusetzen und gemeinsam wirken zu lassen...

(CS I/6: 360, 2. Abs./R 16, letzter Abs.)

„Finde eine Form des Zusammenschlusses, die mit ihrer ganzen Kraft die Person und das Vermögen jedes einzelnen Mitglieds verteidigt und schützt und durch die doch jeder, indem er sich mit allen vereinigt, nur sich selber gehorcht und genauso frei bleibt wit zuvor."

(CS I/6: 260, 4. Abs./R 17, 2. Abs. Mitte) Schließlich gibt sich jeder, da er sich allen gibt, niemandem, und da kein Mitglied existiert, über das man nicht das gleiche Recht erwirbt, das man ihm über sich einräumt, gewinnt man den Gegenwert für alles, was man aufgibt, und mehr Kraft, um zu bewahren, was man hat.

(CS I/6: 3. Abs./R 18, 2. Abs.)

Gemeinsam stellen wir alle, jeder von uns seine Person und seine ganze Kraft unter die oberste Richtschnur des Gemeinwillens; und wir nehmen, als Körper, jedes Glied als untrennbaren Teil des Ganzen auf.

(CS I/6: 361, 4. Abs./R 18, 3. Abs.) 